\title{
Article
}

\section{Mycosphere Essays 7. Ganoderma lucidum - are the beneficial anti- cancer properties substantiated?}

\author{
Hapuarachchi KK ${ }^{1,3,4}$, Wen TC ${ }^{1}$, Jeewon $\mathrm{R}^{5}$, Wu XL ${ }^{6}$, Kang $\mathrm{JC}^{1}$ and Hyde \\ $\mathrm{KD}^{2,3,4}$ \\ ${ }^{1}$ The Engineering Research Center of Southwest Bio-Pharmaceutical Resource Ministry of Education, Guizhou \\ University, Guiyang 550025, Guizhou Province, China \\ ${ }^{2}$ Key Laboratory for Plant Diversity and Biogeography of East Asia, Kunming Institute of Botany, Chinese Academy of \\ Sciences, 132 Lanhei Road, Kunming 650201, China \\ ${ }_{3}$ Center of Excellence in Fungal Research, and ${ }^{4}$ School of Science, Mae Fah Luang University, Chiang Rai 57100 , \\ Thailand \\ ${ }^{5}$ Department of Health Sciences, Faculty of Science, University of Mauritius, Mauritius, \\ 80837 \\ ${ }^{6}$ Guizhou Academy of Sciences, Guiyang, 550009, Guizhou Province, China
}

Hapuarachchi KK, Wen TC, Jeewon R, Wu XL, Kang JC, Hyde KD - Mycosphere Essays 7: Ganoderma lucidum - are the beneficial anti-cancer properties substantiated? Mycosphere 7(3), 305-332, Doi 10.5943/mycosphere/7/3/6

\begin{abstract}
Ganoderma lucidum is a popular medicinal mushroom that has been used particularly in China, Japan and Korea for millennia to improve longevity and health. Research on various metabolic activities of $G$. lucidum have been performed in both in vitro and in vivo studies. There are a vast number of publications that show the abundance and variety of biological actions triggered by the primary metabolites of G. lucidum such as polysaccharides, proteins and triterpenes. However, it is debatable whether G. lucidum is a food supplement for health maintenance or is a therapeutic "drug" for medical purposes. There has been no report of human trials using G. lucidum as a direct control agent for cancers, however, some evidence showing the usage of G. lucidum as potential supplements for cancer patients and a small number of preclinical trials have suggested that it carries promising anti-cancer and immunomodulatory properties. In this review, the beneficial anti-cancer properties of G. lucidum, the evidence for medicinal uses and secondary metabolites, and the effects on human cancer are discussed. G. lucidum and related products can be used as a therapeutic drug, but more direct scientific evidence should be made available in the future. The efficiency of G. lucidum in clinical treatments can be proven by systematic translational research programs using, only standardized preclinically evaluated and biologically active $G$. lucidum extracts in alternative treatments. Hence, studies on G. lucidum should focus on improving methods and further clinical research on human subjects should be performed with more scientific reproducibility. Furthermore research should target pharmacologically active constituents of $G$. lucidum that contribute to positive immune responses, as well as the mode of action of G. lucidum at the molecular level at target organs.
\end{abstract}

Key words - Anti-cancer activity - clinical evidence - Ganoderma lucidum - Lingzhi 


\section{Introduction}

Lingzhi is the Chinese name, mainly applied to Ganoderma lucidum (Curtis) P. Karst. a woody polypore (Basidiomycota) that has been widely used in China for medicinal purposes for more than two thousand years (Sliva 2006). G. lucidum was introduced by Curtis (1871) based on material from Peckham, London, UK and the epithet was sanctioned by Fries (1821). G. lucidum has previously been treated as synonyms of Boletus lucidus (Fr.), Polyporus lucidus Curtis (Fr.), Polyporus polychromus Curtis (Fr.), G. polychromum Curtis (Fr.), G. sessile Curtis (Fr.) and Fomes lucidus (Curtis) Sacc. (Siwulski et al. 2015). Patouillard (1907) reported G. lucidum from China for the first time and Teng (1934) described collections of G. lucidum from different regions in China (Wang et al. 2012). Liu (1974) compiled a monograph of Traditional Chinese Medicinal Fungi, and he described G. lucidum as "Lingzhi" in his book. Since then, G. lucidum was accepted as the scientific binomial of "Lingzhi" in many reports on Chinese edible and medicinal mushrooms (Ying et al. 1987, Mao 1998, Dai et al. 2009). For over a century, "Lingzhi", the highly prized medicinal mushroom in East Asia, has been assigned to G. lucidum, a species originally described from Europe (Cao et al. 2012). Hawksworth (2005) suggested to conserve the name G. lucidum to an Asian type and introduce a new name for the European species (Yang \& Feng 2013). Wang et al. (2012) proposed the name, 'G. lucidum' as used for the Chinese species as incorrect and should be corrected to G. sichuanense. The validity of G. lingzhi and G. sichuanense has been recently debated. Wang \& Yao (2009) proposed that G. sichuanense can represent ' $G$. lucidum' in China. With the aid of molecular phylogeny, Wang et al. (2009) divided Asian specimens classified as $G$. lucidum into two clades; both clades were separated from the European G. lucidum. One clade, composed of tropical collections, represented G. multipileum, while the other clade is undescribed. As G. sichuanense had previously been described, Wang et al. (2009) proposed this name for $G$. lucidum in China. In parallel, Cao et al. (2012) found that the holotype of G. sichuanense was not conspecific with the unnamed clade, and proposed it as a new species called Ganoderma lingzhi, which was considered to be the most widely cultivated species in China. Yao et al. (2013) proposed $G$ sichuanense and $G$. lingzhi as synonymous based on morphological data from an epitype of $G$. sichuanense. However Zhou et al. (2014) again challenged this opinion, with G. lingzhi and G. sichuanense being an independent and taxonomically valid species by stressing that species types depends on geographical distributions. G. lucidum therefore is incorrectly recorded in China, and around the world. Hapuarachchi et al. (2015) reported G. lucidum sensu lato as a species complex whose taxonomy has been confused throughout history and is still debatable. Ryvarden \& Johansen (1980) treated all names of the G. lucidum complex as the "G. lucidum group" because of the lack of specific morphological criteria to name species in this complex. Based on phylogeny by sequences of ITS, tef1a, rpb1 and rpb2 from worldwide samples, Zhou et al. (2015) made a systematical study on the G. lucidum species complex and demonstrated 13 species with color photos However the Chinese "Lingzhi" has continuously been referred to as G. lucidum in monographs of Ganodermataceae in China (Hapuarachchi et al. 2015). G. lucidum has been widely used for naming the commercialized "Lingzhi" products in the worldwide mushroom industry, since it has numerous health benefits (Lai et al. 2004). Hence, in this review we assigned the general name of G. lucidum "Lingzhi", as applied in the previous publications and discuss the beneficial anti-cancer properties, the evidence for medicinal uses, secondary metabolites, and the effects on human cancer.

Ganoderma lucidum is thought to be widely distributed in both tropical and temperate geographical regions, growing as a pathogen or saprotroph on a wide variety of hardwoods (Dai et al. 2007, Dong \& Han 2015). It has large, perennial, and woody basidiocarps (Dong \& Han 2015). Ganoderma lucidum is widely used in Traditional Chinese Medicine and food products as it is believed to increase human longevity and maintenance of vivacity (Sliva 2006). Lingzhi is viewed as the "herb of spiritual potency" or "mushroom of immortality", and symbolizes sanctity, success, goodness and longevity (De Silva et al. 2012a). Chinese herbal medicine has been practiced for thousands of years and is used increasingly in Western countries in conjunction with or in place of allopathic medicine (Yuen \& Gohel 2005). G. lucidum is not used in cooking because of its bitter 
taste and a wooden texture (Chang 1996), however it is consumed in various forms. In recent decades, much attention has been placed on G. lucidum and many studies have reported that a vast number of pharmacological compounds are produced by G. lucidum (Paterson 2006). The pharmacological effect of G. lucidum is based on its strong immune-modulating action and immune potential capability, which supports and enhances the overall immune function (Singh et al. 2015). Studies have revealed that $G$. lucidum contains approximately 400 different bioactive compounds (Dong \& Han 2015) with polysaccharides, steroids and triterpenes as the major groups (Boh et al.

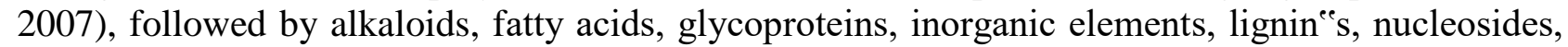
nucleotides, peptides, phenols, proteins, sterols and vitamins (Paterson 2006, Li et al. 2013). Bioactive compounds from G. lucidum hold an enormous structural and chemical diversity which is incomparable to any synthetic library ( $\mathrm{Li}$ et al. 2013). These bioactive constituents are reported to be responsible for the anti-cancer, anti-inflammatory, anti-tumor, anti-oxidant, immunomodulatory, immunodeficiency, anti-diabetic, anti-viral, anti-bacterial, anti- fungal, anti-hypertensive, antiatherosclerotic, anti-aging, anti-androgenic, anti-hepatotoxic, radical scavenging property, neuroprotection, sleep promotion, cholesterol synthesis inhibition, hypoglycemia, inhibition of lipid peroxidation/oxidative DNA damage, hepatoprotective properties, maintenance of gut health, prevention of obesity, and stimulation of probiotics (Paterson 2006, Cao et al. 2013, De Silva et al. 2012a, b, De Silva et al. 2013, Baby et al. 2015, Bishop et al. 2015, Liu et al. 2015). G. lucidum has been used as a functional food to prevent and treat many immunological diseases, such as anorexia, arthritis, asthma, bronchitis, cardiovascular problems, constipation, diabetes, dysmenorrhea, gastritis, hemorrhoids, hepatitis hypercholesterolemia, hypertension, insomnia, lupus erythematosis, migraine, nephritis, neurasthenia, neoplasia and tumorigenesis (Liu et al. 2002, Paterson 2006, Wang et al. 2012, Tan et al. 2015). The anti-cancer effect of G. lucidum is a widely studied topic (Liu et al. 2015). Yu \& Shen (2003) reported Lingzhi (G. lucidum) was cultivated successfully for the first time in 1969 in China and currently, the annual sale of products derived from G. lucidum is estimated to be more than US\$2.5 billion in Asian countries, including China, Japan, and South Korea ( $\mathrm{Li}$ et al. 2013). G. lucidum products, from different parts of its fruiting bodies, mycelia or spores, are sold in the form of coffee, powder, tea, dietary supplements, spore products, drinks, syrups, tooth pastes, soaps and lotions and have been commercialized as food and drug supplements which enhance the body's immune system and improve metabolic functions (Chang \& Buswell 1999, Lai et al. 2004, Singh et al. 2013). Although G. lucidum has many reported medicinal properties, the mechanisms of their actions have been poorly described. Improved research techniques that can demonstrate the health benefits of G. lucidum more effectively are now becoming increasingly possible (Bishop et al. 2015). Secondary metabolites have been isolated from various Ganoderma species with 240 isolated from G. lucidum alone (112 C30 Ganoderic acids, 55 other C30 lanostanes, 27 C27 Lucidenic acids, 18 other C27 lanostanes, three C24 lanostanes, one meroterpenoid, 23 steroids and one benzofuran). Since "Lingzhi" has been used as a traditional Chinese medicine for over two millennia, it has become an important research topic and is very popular in China. However, it is not clear if the claimed benefits of taking "Lingzhi" in various forms are substantiated. In the following part of this review, we discuss various bioactive compounds produced by the mushroom and their anti-cancer activities. We present and discuss experimental evidence in connection with "Lingzhi" and its beneficial medicinal properties.

\section{Ganoderma lucidum as an anti-cancer agent}

Cancer is a worldwide health problem and latest statistics of the World Cancer Research Fund International, reveals there are an estimated 14.1 million cancer cases around the world in 2012, and this number is expected to increase to 24 million by 2035 (http://www.wcrf.org/). There were 1, 665,540 new cancer cases and 585,720 cancer deaths estimated in the United States in 2014, and certain malignant tumors are a major cause of death (Cheng \& Sliva 2015). Cancer is an abnormal growth of cells which proliferates in an uncontrolled way and, in some cases, metastasizes or spreads (Borchers et al. 2004, Zaidman et al. 2005, Ruddon 2007, NCI 2011, De 
Silva et al. 2013). Uncontrolled cell proliferation can be induced by many factors, including chemical, physical, or biological agents (Anand et al. 2008, NCI 2011, WHO 2011). Chemotherapy and radiotherapy are two commonly used conventional treatments for cancer patients, however, a variety of adverse events are associated with these two treatments. Hence, oncological practices have looked for alternative cancer medications over the past few decades (Jin et al. 2012). Cyclephase-specific anti-cancer drugs have either cytotoxic or cytostatic activities. Hence, cytotoxic drugs, including most of the chemotherapeutics, are toxic to cells and usually cause DNA damage, resulting in programmed cell death (apoptosis and autophagy) (Cheng \& Sliva 2015). Cytostatic drugs however, can stop the rapid proliferation of cancer cells, instead of killing them directly (Cheng \& Sliva 2015). Recent laboratory and preclinical studies in vitro and in vivo, concluded that the anti-cancer activity of Ganoderma lucidum can be attributed to a variety of different mechanisms. The direct cytotoxic and anti-angiogenesis mechanisms of G. lucidum have been established by in vitro and animal studies (Yuen \& Gohel 2005). G. lucidum has been positively demonstrated as a treatment for most human and murine cancer cell lines tested specifically for lung, liver, breast, prostate, sarcoma, colon, bladder, cervix, and leukemia lines. Furthermore, studies on hepatoma and sarcoma cells with G. lucidum, have been extended to in vivo animal models which resulted in inhibition of tumor growth and metastasis (Yuen \& Gohel 2005). Jin et al. (2016) demonstrated that there is insufficient evidence available to justify the use of G. lucidum as a first-line treatment for cancer. It is still debatable whether G. lucidum prolongs long-term cancer survival. G. lucidum, however could be administered as an alternative supplement to conventional treatment because it has potential to enhance tumor response and stimulate host immunity. Furthermore, G. lucidum was generally well tolerated by most participants with only a small number of minor adverse effects and no major toxicity. Although there have been small number of reports on the minor effects of G. lucidum, the use of its extract should be cautious, especially after thorough consideration of cost benefit and patient preference. Jin et al. (2012) found that patients who used G. lucidum extracts in their anti-cancer treatments were 1.27 times more likely to respond to chemotherapy or radiotherapy. However, the study failed to demonstrate significant effects on tumor shrinkage when $G$. lucidum extract was used alone. G. lucidum extracts could stimulate host immune functions by considerably increasing CD3, CD4 and CD8 lymphocyte percentages, however natural killer (NK) cell activity, which is suggested to be an indicator of self-defense against tumour cells, was marginally elevated. Patients were found to have a relatively better quality of life after the treatment with G. lucidum extract. Minor side effects were reported following G. lucidum treatment, including nausea and insomnia. Tables 1 and 2 show the cytotoxic effects of the concentration that inhibits $50 \%$ of cell proliferation (IC 50) and the lethal dose that causes $50 \%$ of cell death (LD 50) varied from 1 to $5,000 \mu \mathrm{g} / \mathrm{ml}$ with various components of $G$. lucidum. Furthermore, pure compounds of G. lucidum showed cytotoxic activity at very low concentrations. G. lucidum can exhibit anti-cancer activity on different types of cancers from different origins. Some G. lucidum products on the market are labeled as "immunomodulating" agents, which act potentially as a supplement to support cancer patients, but are substantiated by only two clinical trials (Yuen \& Gohel 2005). Some evidence presented by some research groups and the mechanisms of anti-cancer agents are not explicitly documented (Yuen \& Gohel 2005). There are some in vitro and in vivo animal studies that confirm G. lucidum has anti-cancer activity, but further research is essential, with human trials on direct anti-cancer activity. To date G. lucidum appears to be beneficial to cancer patients and can possibly become a new anti-cancer agent in the future assuming satisfactory clinical trials are performed.

\section{Active anti-cancer compounds of Ganoderma lucidum}

\section{Triterpenoids}

Triterpenes isolated from Ganoderma lucidum species have been identified as potential anticancer agents and have structural similarity to steroid hormones and exhibit a broad spectrum of anti-cancer properties (Paterson 2006, Cheng et al. 2010, De Silva et al. 2012a, Wu et al. 2012, 
Cheng \& Sliva 2015). Wu et al. (2013) have been identified fifteen triterpenoids and furthermore two new triterpenoids reported by Zhao et al. (2015) from fruiting bodies of fungus G. lucidum. Triterpenoids such as Ganoderic acids, lucidimols, ganodermanondiol, ganoderiol $\mathrm{F}$ and ganodermanontriol, exert cytotoxic effects on various cancer cells (Chen \& Chen 2003, Sliva 2003, Chang et al. 2006, Tang et al. 2006a, Weng \& Yen 2010, Chin et al. 2011, De Silva et al. 2013). Cytotoxic triterpenoids inhibit human cervical cancer cells, and act as an alternative dietary approach for the prevention of colitis associated cancer (Cheng et al. 2010, Xu et al. 2010, De Silva et al. 2013). Ganoderic acids have been found to have direct cancer cell cytotoxicity on a wide variety of cancer cell lines, such as murine Lewis lung carcinoma (LLC) and Meth-A, and many of them have been suggested to counter angiogenesis and metastasis (Min 2000). Yue et al. (2008) reported that treatments with $G$. lucidum triterpenes regulated expression of 14 proteins in human cervical carcinoma cells. These proteins play important roles in cell proliferation, cell cycle, oxidative stress, and apoptosis. G. lucidum triterpenes induced sensitization of cells to the chemotherapeutic doxorubicin by increasing oxidative stress, DNA damage, and apoptosis (Cheng \& Sliva 2015). G. lucidum triterpene extract suppressed proliferation of human colon cancer cells and also inhibited tumor growth in a xenograft model, which was associated with the cell cycle arrest at the G0/G1 phase and induction of the programmed cell death Type II autophagy (Cheng \& Sliva 2015). Jiang et al. (2008) reported that Ganoderic acid A can suppress proliferation and colony formation of Cdk4 in breast cancer cells through inhibition of transcription factors AP-1 and $\mathrm{NF}-\kappa \mathrm{B}$, which result in the down-regulation of expression of Cdk4. Furthermore, Ganoderic acid A inhibited phosphorylation or activation of transcription factor STAT3 which results an enhanced sensitivity of hepatoma cells to cisplatin. Apoptosis is induced by Ganoderic acid A in lymphoma cells through caspase-3 and caspase-9, and enhanced HLA class II-mediated antigen presentation and CD4+ T-cell recognition (Radwan et al. 2015). Ganoderic acid DM inhibited cell proliferation, induced the DNA damage, cell cycle arrest at the G1 phase, and apoptosis in human breast cancer cells and further improve the antigen presentation and CD4+ T-cell recognition in melanoma cells (Radwan et al. 2015). The growth of hepatoma cells is inhibited by triterpene enriched extracts from G. lucidum via suppressing protein kinase $\mathrm{C}$ and activating mitogen-activated protein kinases (Lin et al. 2003). Ganoderic acid T (12) induces apoptosis of metastatic lung tumor cells, through an intrinsic pathway related to mitochondrial dysfunction (Tang et al. 2006b). Semisynthetic modification of Ganoderic acid $\mathrm{T}$ produced more effective anti-cancer agents (Liu et al. 2012c). Cytotoxicity of a Ganoderic acid fraction called GA-Me has been tested on human colon cancer cells (Chen et al. 2008) and activation of the intrinsic mitochondria-dependent apoptotic pathway was identified and it was found that GA-Me is a potential natural apoptosis inducing agent for human colon tumors (Zhou et al. 2011). Ganoderic acid DM effectively inhibits cell proliferation and colony formation in MCF-7 human breast cancer cells. It exerted its anti-proliferative effect by inducing cell cycle (G1) arrest and apoptosis in MCF7 cells (Liu et al. 2012d, Wu et al. 2012, De Silva et al. 2013). Lucidenic acids A, B, C, and N have been isolated from fruiting bodies of $G$. lucidum and the extracts have anti-invasive effects on hepatoma cells, owing to extraordinary highs

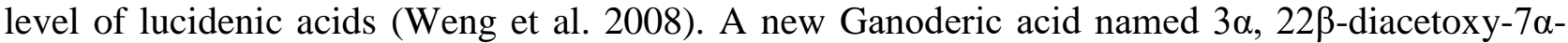
hydroxy-5 $\alpha$ - lanosta-8, 24 E-dien-26-oic acid was isolated from $G$. lucidum mycelia with substantial cytotoxic activity (Li et al. 2013). G. lucidum AF (antlered form of G. lucidum) contains a higher amount of triterpenes than normal G. lucidum giving potent immunomodulatory and antitumor effects (Nonaka et al. 2008, Watanabe et al. 2011, De Silva et al. 2013). Two new lanostane

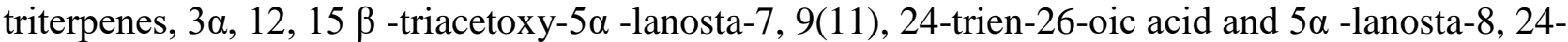
diene-26, 27-dihydroxy-3, 7-dione were isolated from fruiting bodies of a Vietnamese strain of $G$ lucidum having potential cytotoxic activities against human non-small cell lung adenocarcinoma (A549), breast adenocarcinoma (MCF-7), and prostatic small cell carcinoma (PC-3) (Nguyen et al. 2015). Treatment of cancer cells with G. lucidum extracts causes down-regulation of cell cycle associated proteins resulting in cell cycle arrest (Jedinak et al. 2011, Sliva et al. 2012, WHO 2014, Wu et al. 2013). Important triterpenoids, extracts, actions, IC 50 and LD50 values of G. lucidum, are listed in Tables 1 and 2. 
Table 1 Anti-cancer activities of some important triterpenoids and Ganoderma lucidum extracts with IC 50 values

\begin{tabular}{|c|c|c|c|c|c|}
\hline Triterpene & Cancer cells & Mechanism of action & Target & $\begin{array}{l}\mathrm{IC} \mathrm{50} \\
(\mu \mathrm{g} / \mathrm{ml})\end{array}$ & References \\
\hline \multirow[t]{3}{*}{ Ganoderic acid $\mathrm{A}$} & Breast: MDA MB-231 & $\begin{array}{l}\text { Inhibition of cell } \\
\text { proliferation and } \\
\text { colony formation, } \\
\text { suppression } \\
\text { of cell adhesion, migration, } \\
\text { invasion }\end{array}$ & $\begin{array}{l}\text { NF-к B, AP-1, Cdk4, u } \\
\text { PA, uPAR }\end{array}$ & 291 & Jiang et al. 2008 \\
\hline & Liver: Hep G2 & $\begin{array}{l}\text { Increased sensitivity to } \\
\text { cisplatin }\end{array}$ & STAT3 & N/A & Yao et al. 2012 \\
\hline & $\begin{array}{l}\text { Lymphoma: } \\
\text { NALM-6 }\end{array}$ & Induction of apoptosis & $\begin{array}{l}\text { Caspase-3, } \quad-9, \quad \text { BIM, } \\
\text { BAX }\end{array}$ & N/A & Radwan et al. 2015 \\
\hline \multirow[t]{2}{*}{ Ganoderic acid DM } & $\begin{array}{l}\text { Breast: MCF-7, MDA- } \\
\text { MB-231 }\end{array}$ & 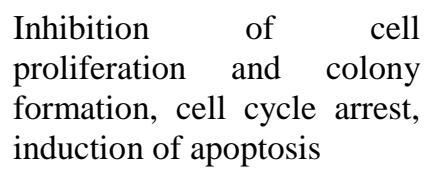 & $\begin{array}{l}\text { Cdk2-4, cyclin D1, pRb, } \\
\text { c-Myc, PARP }\end{array}$ & 352 & Wu et al. 2012 \\
\hline & Melanoma: J3, HT-144 & $\begin{array}{l}\text { Induction of apoptosis and } \\
\text { autophagy }\end{array}$ & Caspase-3, -9 , Bax & N/A & Hossain et al. 2012 \\
\hline Ganoderic acid $\mathbf{H}$ & Breast: MDA-MB-231 & $\begin{array}{l}\text { Inhibition of cell } \\
\text { proliferation and } \\
\text { colony formation, } \\
\text { suppression } \\
\text { of cell adhesion, migration, } \\
\text { invasion }\end{array}$ & $\begin{array}{l}\text { NF-B, AP-1, Cdk4, u } \\
\text { PA, uPAR }\end{array}$ & N/A & Jiang et al. 2008 \\
\hline Ganoderic acid Jc & $\begin{array}{l}\text { Leukemia: HL- } 60 \\
\text { Breast: MCF-7 }\end{array}$ & Cytotoxic & N/A & N/A & Liu et al. 2012 \\
\hline Ganoderic acid Mc & $\begin{array}{l}\text { Cervix: HeLa } \\
\text { Lung: 95D }\end{array}$ & Cytotoxic & N/A & N/A & Li et al. 2013 \\
\hline \multirow[t]{3}{*}{ Ganoderic acid - Me } & Breast: MDA-MB-231 & $\begin{array}{l}\text { Inhibition } \\
\text { proliferation, angiogenesis } \\
\text { and invasion. Induction of } \\
\text { apoptosis, }\end{array}$ & $\begin{array}{l}\text { NF-кB, с-Myc, cyclin } \\
\text { D1, Bcl-2, MMP-9, } \\
\text { VEGF, IL-6, -8 }\end{array}$ & N/A & Li et al. 2012 \\
\hline & Lung: 95D & $\begin{array}{l}\text { inhibition of adhesion and } \\
\text { migration of cells }\end{array}$ & MMP-2/9 & N/A & Chen et al. 2008 \\
\hline & LLC implanted C57BL/6 & Inhibition of lung metastasis & $\begin{array}{l}\text { IL-2, and IFN-c, NK } \\
\text { cells }\end{array}$ & N/A & Weng \& Yen 2010 \\
\hline \multirow[t]{2}{*}{ Ganoderic acid Mf/S } & Cervix: He La & $\begin{array}{l}\text { Cell cycle arrest, induction } \\
\text { of apoptosis }\end{array}$ & Caspase-3, -9, Bax & N/A & Liu et al. 2011 \\
\hline & Lung: 95D & Cytotoxic & N/A & N/A & \\
\hline Ganoderic acid T; & Cervix: $\mathrm{He} \mathrm{La}$ & Inhibition & NF-кB, MMP-9, i NOS & 281 & Liu et al. 2012 \\
\hline
\end{tabular}




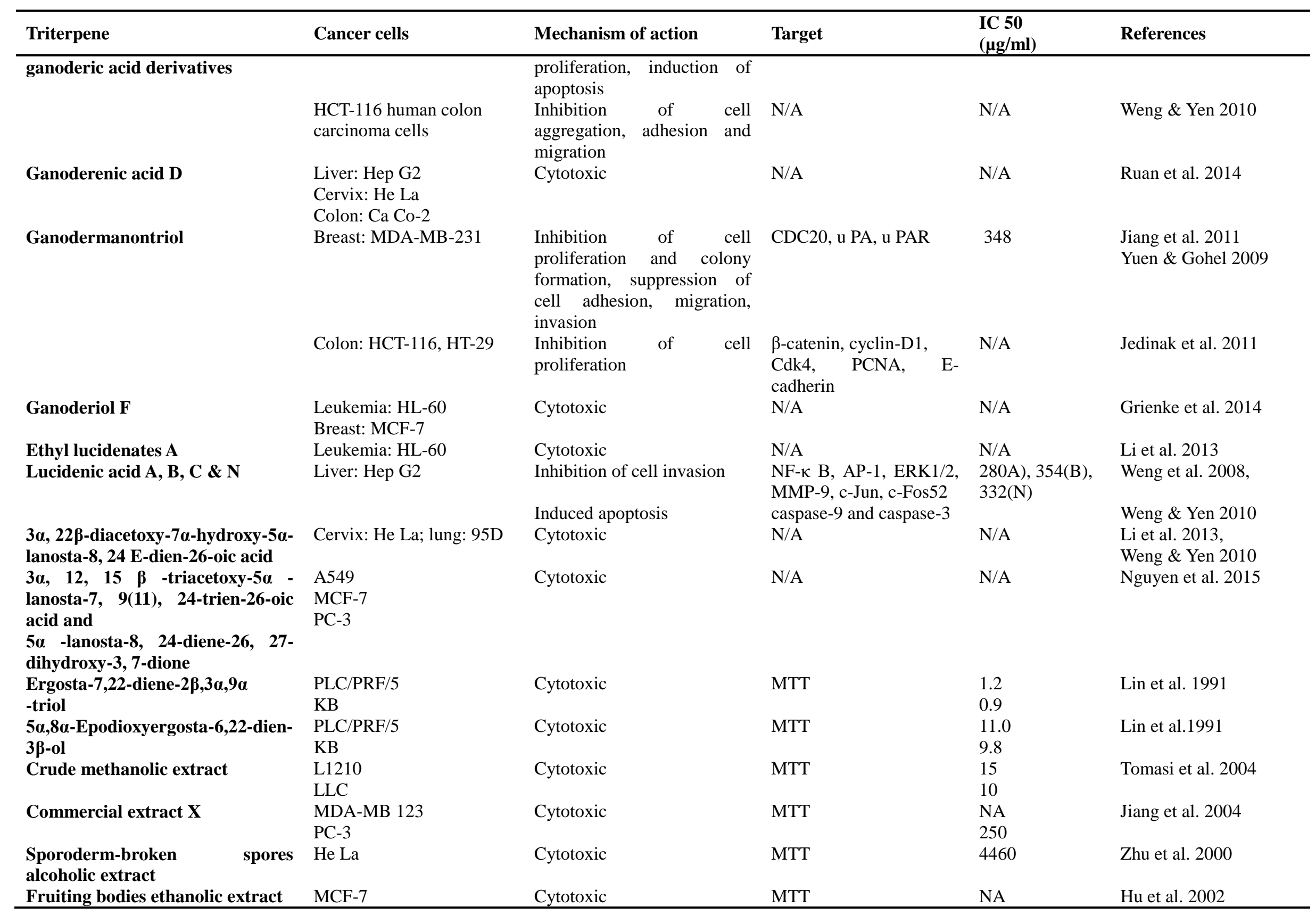




\begin{tabular}{|c|c|c|c|c|c|}
\hline Triterpene & Cancer cells & Mechanism of action & Target & $\begin{array}{l}\text { IC 50 } \\
(\mu \mathrm{g} / \mathrm{ml})\end{array}$ & References \\
\hline & MTC-11 & & MTT & 129.3 & Lu et al. 2004 \\
\hline & & & HTIA & 113.0 & \\
\hline \multirow{2}{*}{ Fruiting bodies water extract } & HUC-PC & Cytotoxic & MTT & 509.0 & Lu et al. 2004 \\
\hline & & & HTIA & 990.0 & \\
\hline \multirow[t]{2}{*}{ spores ethanolic extract } & HUC-PC & Cytotoxic & MTT & 274.7 & Lu et al. 2004 \\
\hline & & & HTIA & 234.0 & \\
\hline \multirow{2}{*}{ Spores water extract } & HUC-PC & Cytotoxic & MTT & 365.0 & Lu et al. 2004 \\
\hline & & & HTIA & 465.0 & \\
\hline
\end{tabular}

A549: human non-small cell lung adenocarcinoma; NA: not available; HTIA: 3H thymidine incorporation assay; HUC-PC: Human uroepithelial cell line; MTC-11: low-grade bladder cancer cell line; MTT: tetrazolium method; MCF-7: breast adenocarcinoma; PC-3: prostatic small cell carcinoma; IC50-concentration that inhibits 50\% of cell proliferation (Yuen \& Gohel 2005).

Table 2 Anti-cancer activities of Ganoderma lucidum triterpenes and extracts with LD 50 values

\begin{tabular}{|c|c|c|c|c|}
\hline Ganoderma lucidum product/extract/pure compound & Cell Line & $\mathrm{LD50}(\mu \mathrm{g} / \mathrm{ml})$ & Method & Reference \\
\hline Triterpene-enriched mycelial ethanolic extract & Huh-7 & 450 & ACP 32 & Lin et al. 2003 \\
\hline Sporoderm-broken spores alcoholic extract & $\mathrm{He} \mathrm{La}$ & 4,700 & TB 30 & Zhu et al. 2000 \\
\hline Culture broth of mycelia & Hep3B & NA & TB & Chung et al. 2003 \\
\hline Ganoderic acid $\mathbf{G} 18$ & Meth-A & 6.8 & SRB & Min et al. 2000 \\
\hline Ganoderic acid $\gamma$ & NA & 15.6 & NA & Yuen \& Gohel 2009 \\
\hline Ganoderic acid $\varepsilon$ & NA & 12.2 & NA & Yuen \& Gohel 2009 \\
\hline \multirow[t]{2}{*}{ Ganoderic acid $\theta$} & NA & 5.7 & NA & Yuen \& Gohel 2009 \\
\hline & LLC & 15.2 & & \\
\hline Ganoderic acid C1 & & 17 & NA & Yuen \& Gohel 2009 \\
\hline Ganoderiol F 6 & Meth-A & 4.4 & NA & Yuen \& Gohel 2009 \\
\hline Ganodermanontriol & LLC & 9.6 & NA & Yuen \& Gohel 2009 \\
\hline Ganolucidic acid A & NA & 15.5 & NA & Yuen \& Gohel 2009 \\
\hline Lucidenic acid $\alpha$ & NA & 17.8 & NA & Yuen \& Gohel 2009 \\
\hline Lucidumol A & Meth-A & 2.3 & NA & Yuen \& Gohel 2009 \\
\hline \multirow[t]{2}{*}{ Lucidumol B } & LLC & 8.5 & NA & Yuen \& Gohel 2009 \\
\hline & & 16.6 & & \\
\hline \multirow[t]{6}{*}{ Ganodermanondiol } & Meth-A & 12.5 & NA & Gao et al. 2002 \\
\hline & NA & 3.4 & NA & \\
\hline & NA & 9.2 & SRB & \\
\hline & LLC & 14 & & \\
\hline & $\mathrm{T}-47 \mathrm{D}$ & 4.7 & & \\
\hline & S-180 & 11 & & \\
\hline Ganodermnonol & $\mathrm{T}-47 \mathrm{D}$ & 10 & NA & Gao et al. 2002 \\
\hline
\end{tabular}




\begin{tabular}{|c|c|c|c|c|}
\hline & NA & 4.8 & & \\
\hline & Meth-A & 2.8 & & \\
\hline Ganodermadiol & NA & 10.3 & NA & Yuen \& Gohel 2009 \\
\hline Lucialdehyde A & NA & 10.4 & NA & Yuen \& Gohel 2009 \\
\hline \multirow[t]{3}{*}{ Lucialdehyde C } & LLC & 10.7 & NA & Yuen \& Gohel 2009 \\
\hline & $\mathrm{T}-47 \mathrm{D}$ & 4.7 & & \\
\hline & S-180 & 7.1 & & \\
\hline \multirow[t]{4}{*}{ Lucialdehyde B } & NA & 4 & NA & Yuen \& Gohel 2009 \\
\hline & NA & NA & & \\
\hline & T-47D LLC & 15 & & \\
\hline & & 14.3 & & \\
\hline
\end{tabular}

Table 3 Main polysaccharides from Ganoderma lucidum with anti-cancer activities

\begin{tabular}{|c|c|c|c|c|}
\hline Polysaccharides & $\begin{array}{l}\text { Major } \\
\text { monosaccharides }\end{array}$ & Cancer cell type & Mechanism of action & References \\
\hline $\begin{array}{l}\text { Se } \\
\text { entiched } \quad G . \quad \text { lucidum } \\
\text { polysaccharide) }\end{array}$ & $\begin{array}{l}\text { Glucose } \\
\text { mannose }\end{array}$ & Different cancer cells & $\begin{array}{l}\text { Proliferation of different cancer cell lines in vitro } \\
\text { and induces mitochondria-mediated apoptosis }\end{array}$ & Zhang et al. 2011 \\
\hline $\begin{array}{l}\text { G lucidum } \\
\text { polysaccharides fraction } \\
3(\text { F3) }\end{array}$ & $\begin{array}{l}\text { Glucose, } \\
\text { mannose }\end{array}$ & $\begin{array}{l}\text { Human } \\
\text { leukemia THP-1 } \\
\text { cells }\end{array}$ & $\begin{array}{l}\text { Cytotoxic. Induces macrophage-like differentiation } \\
\text { and apoptosis }\end{array}$ & Cheng et al. 2007 \\
\hline LZP-F3 & $\begin{array}{l}\text { Glucose, galactose, } \\
\text { mannose }\end{array}$ & $\begin{array}{l}\text { Bladder urothelial } \\
\text { carcinoma cell N/P (14) and N/As (0.5) }\end{array}$ & $\begin{array}{l}\text { Modulating the expression and activation of } \\
\text { apoptosis correlated proteins }\end{array}$ & Huang et al. 2010 \\
\hline GLIS & Glucose, & Tumor growth cancer cells & $\begin{array}{l}\text { Inhibitory effect on tumor growth and dramatically } \\
\text { enhanced both humoral and cellular immune } \\
\text { functions in a mouse model }\end{array}$ & Zhang et al. 2010 \\
\hline \multirow[t]{2}{*}{ GlPS } & $\begin{array}{l}\text { Fructose, } \\
\text { glucose }\end{array}$ & Tumor growth cancer cells & $\begin{array}{l}\text { Inhibited tumor } \\
\text { growth in a murine sarcoma } 180 \text { model and the } \\
\text { adhesion ability of tumor cells to HUVECs } \\
\text { via the up-regulation } \\
\text { of serum amyloid }\end{array}$ & Zhu et al. 2007 \\
\hline & & & $\begin{array}{l}\text { Immunomodulation } \\
\text { effect (on lymphocytes) and cyclophosphamide } \\
\text { induced immunosuppression }\end{array}$ & \\
\hline
\end{tabular}




\begin{tabular}{|c|c|c|c|c|}
\hline Polysaccharides & $\begin{array}{l}\text { Major } \\
\text { monosaccharides }\end{array}$ & Cancer cell type & Mechanism of action & References \\
\hline \multirow[b]{2}{*}{ GlPP } & \multirow[b]{2}{*}{$\begin{array}{l}\text { Xylose, } \\
\text { Glucose }\end{array}$} & Lung cancer & $\begin{array}{l}\text { in mice } \\
\text { Promoted cancer cells to induce lymphocyte } \\
\text { proliferation and activation }\end{array}$ & \multirow{2}{*}{$\begin{array}{l}\text { Sun et al. } 2011 \\
\text { Sun et al. } 2013 \\
\text { Cao \& Lin } 2006\end{array}$} \\
\hline & & $\begin{array}{l}\text { Human lung cancer } \\
\text { cells }\end{array}$ & $\begin{array}{l}\text { fully or partially reversed lymphocyte suppression } \\
\text { Inhibit the proliferation } \\
\text { of HUVECs by inducing } \\
\text { cell apoptosis and decrease the expression } \\
\text { of secreted VEGF }\end{array}$ & \\
\hline \multirow[t]{2}{*}{ GLPP } & \multirow[t]{2}{*}{ Glucose } & Tumor growth cancer cells & $\begin{array}{l}\text { Inhibited the growth } \\
\text { of inoculated } \\
\text { S180, Heps, and } \\
\text { EAC tumor cells in mice } \\
\text { with an enhanced } \\
\text { host immunofunction, and prevented the } \\
\text { immunosuppression induced by } \\
\text { cyclophosphamide treatment and } 60 \\
\text { Co radiation in mice }\end{array}$ & Pang et al. 2007 \\
\hline & & Gastric cancer & $\begin{array}{l}\text { Reduction of IL- } 6 \text { and TNF- } \alpha \text { levels and increased } \\
\text { concentration of IL-2, IL- } 4 \text {, and IL-10 in serum of } \\
\text { rats with } 65\end{array}$ & Pan et al. 2013 \\
\hline $\begin{array}{l}\text { Ganoderma lucidum } \\
\text { polysaccharide } \\
\text { derivatives (S-GL and } \\
\text { CM-GL) }\end{array}$ & Glucose & NA & $\begin{array}{l}\text { Induced cell cycle arrest at the } \mathrm{G} 2 / \mathrm{M} \text { phase and } \\
\text { suppressed the growth of sarcoma tumor cells in } \\
\text { vitro and in vivo with low toxicity to animals }\end{array}$ & Wang et al. 2009 \\
\hline $\begin{array}{l}\text { Ethanol extracts of } G \\
\text { lucidum }\end{array}$ & Glucose & Human gastric carcinoma cells & Induction of apoptosis & Jang et al. 2010 \\
\hline (LZ- D-7) & Glucose & Tumor & $\begin{array}{l}\text { Activates the B-cells } \\
\text { and acts as immunostimulatory drug }\end{array}$ & Ye et al. 2011 \\
\hline GSG & Glucose & Murine resident peritoneal macrophages & $\begin{array}{l}\text { induce IL- } 6 \text { and TNF- } \alpha \text { secretion } \\
\text { Dectin-1, } \\
\text { MAPKs- and Syk- dependent pathway }\end{array}$ & Guo et al. 2009 \\
\hline
\end{tabular}


Table 4 Pharmacologically active proteins isolated from Ganoderma lucidum

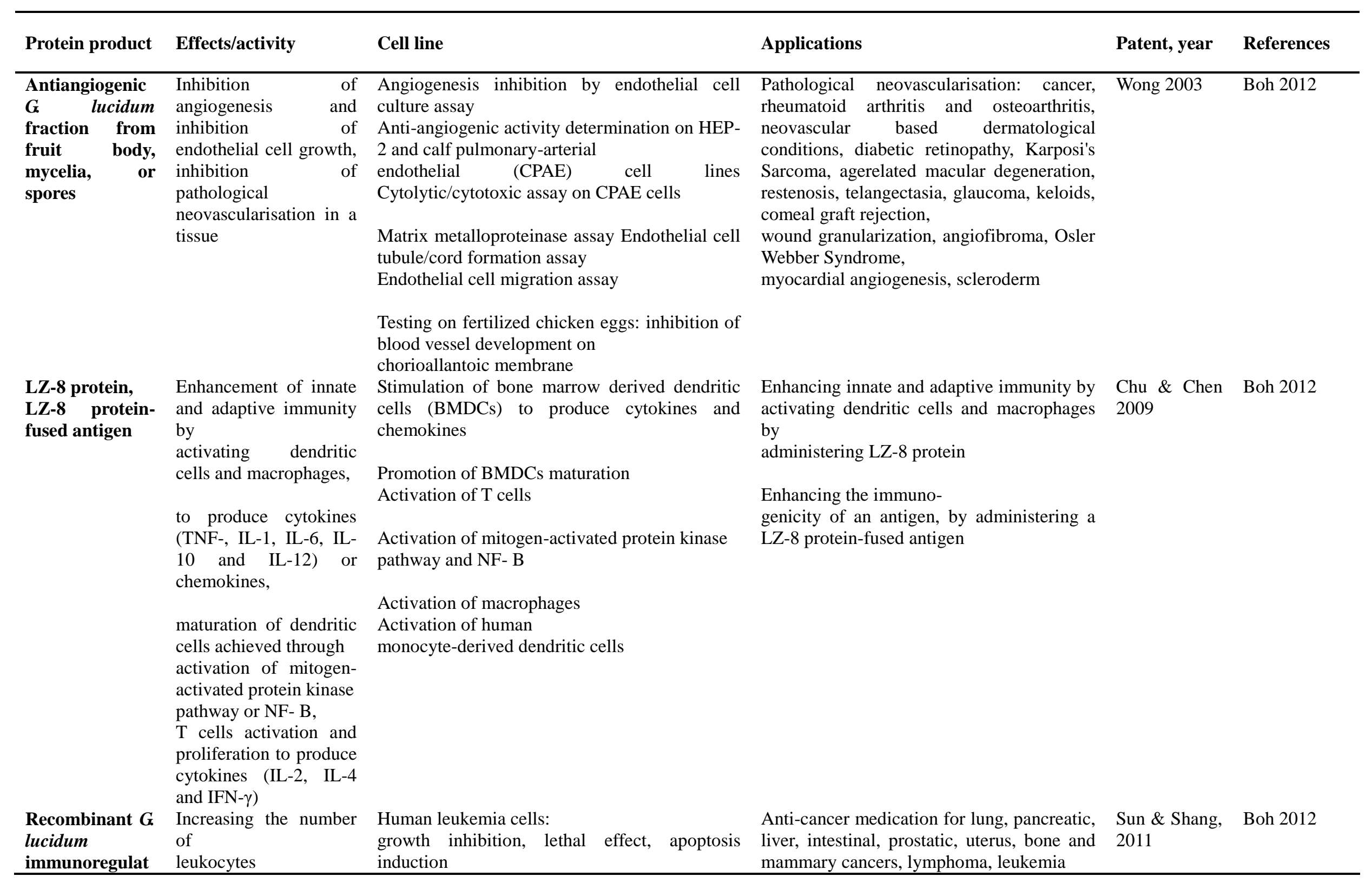




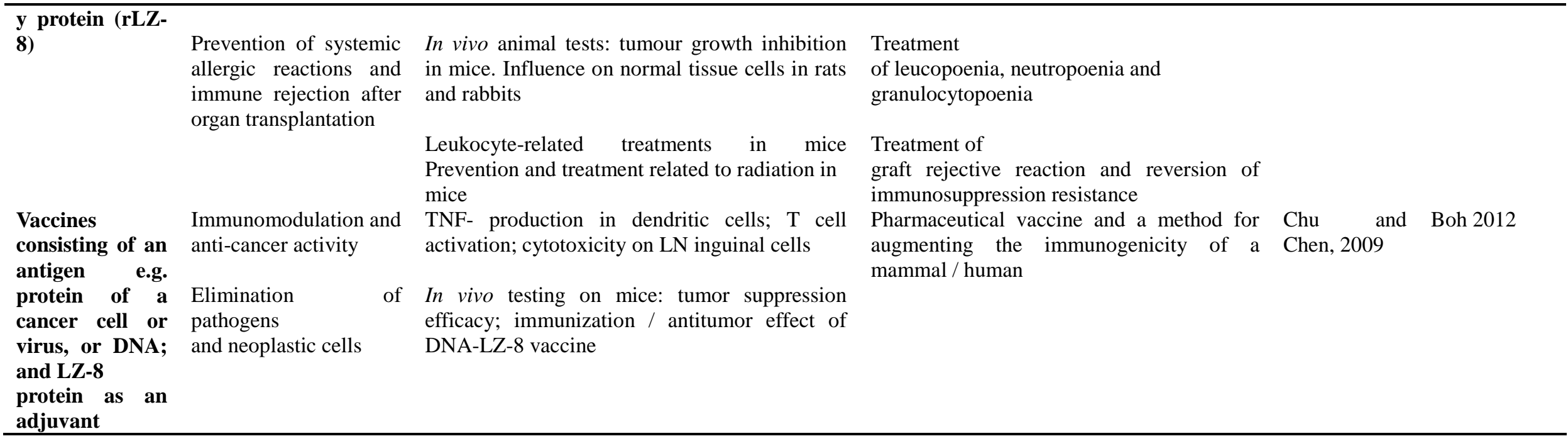

\section{Published medical investigations with Ganoderma lucidum preparations, case studies and clinical trials}

Table 5 Clinical trials carried out with Ganoderma lucidum preparations

\begin{tabular}{|c|c|c|c|c|}
\hline Clinical trial & Cancer type & Dose & Effect & Reference \\
\hline $\begin{array}{l}\text { Non randomized } \\
\text { clinical studies of } 140 \\
\text { cancer patients, all } \\
\text { with metastasis }\end{array}$ & Breast cancer & $\begin{array}{l}6 \mathrm{~g} \text { of } \mathrm{G} . \text { lucidum essence per day for } 6 \\
\text { months }\end{array}$ & $\begin{array}{l}\text { Six patients with breast cancer showed promising } \\
\text { results, a woman over } 50 \text { with } \\
\text { breast cancer metastasized to the lung and after the } \\
\text { treatment her lung tumor disappeared, }\end{array}$ & $\begin{array}{l}\text { Morishige } \\
1988 \\
\text { (Information } \\
\text { taken from Chen \& } \\
\text { Seleen 2007) }\end{array}$ \\
\hline & & $\begin{array}{l}9 \mathrm{~g} \text { per day of } G . \text { lucidum } \\
\text { essence with a high dosage of vitamin } \mathrm{C} \\
\text { for } 2 \text { months, } \\
\text { the dosage } \\
\text { increased later to } 20 \mathrm{~g} \text { per day }\end{array}$ & $\begin{array}{l}\text { cancer patient with metastasis to the bone, has } \\
\text { improved } \\
\text { considerably }\end{array}$ & \\
\hline $\begin{array}{l}\text { A patient diagnosed } \\
\text { with hepatoma treated }\end{array}$ & $\begin{array}{l}\text { Hepatoma (at the portal } \\
\text { vein region of the liver) }\end{array}$ & $\begin{array}{l}\text { High dose of Ganoderma spores } \\
\text { treatment from May to August } 1999\end{array}$ & $\begin{array}{l}\text { Tumor size } 5.1 \times 6.6 \times 7.7 \mathrm{~cm} \text { in May } 1999 \text {, reduced } \\
\text { to } 3.5 \times 3.4 \times 3 \mathrm{~cm} \text { in August, } 1999\end{array}$ & Liu \& Chung 2001 \\
\hline $\begin{array}{l}\text { with } \quad \text { Ganoderma } \\
\text { spores }\end{array}$ & & (Ganoderma spore treatment only) & & \\
\hline $\begin{array}{l}\text { Chinese } G \text { lucidum } \\
\text { essence for } 547 \text { cancer } \\
\text { patients }\end{array}$ & $\begin{array}{l}\text { Medium and late phase } \\
\text { cancer }\end{array}$ & $\begin{array}{l}\text { A continuous } 2-3 \text {-month active long } \\
\text { treatment with a daily dosage of } 4-6 \mathrm{~g} \text { of } \\
\text { G. lucidum essence, further dosage of } 2 \mathrm{~g} \\
\text { per day continuously after the third }\end{array}$ & $\begin{array}{l}\text { Significantly lower death rate of patients in the long- } \\
\text { term treatment }\end{array}$ & Shi \& Quing 2001 \\
\hline
\end{tabular}




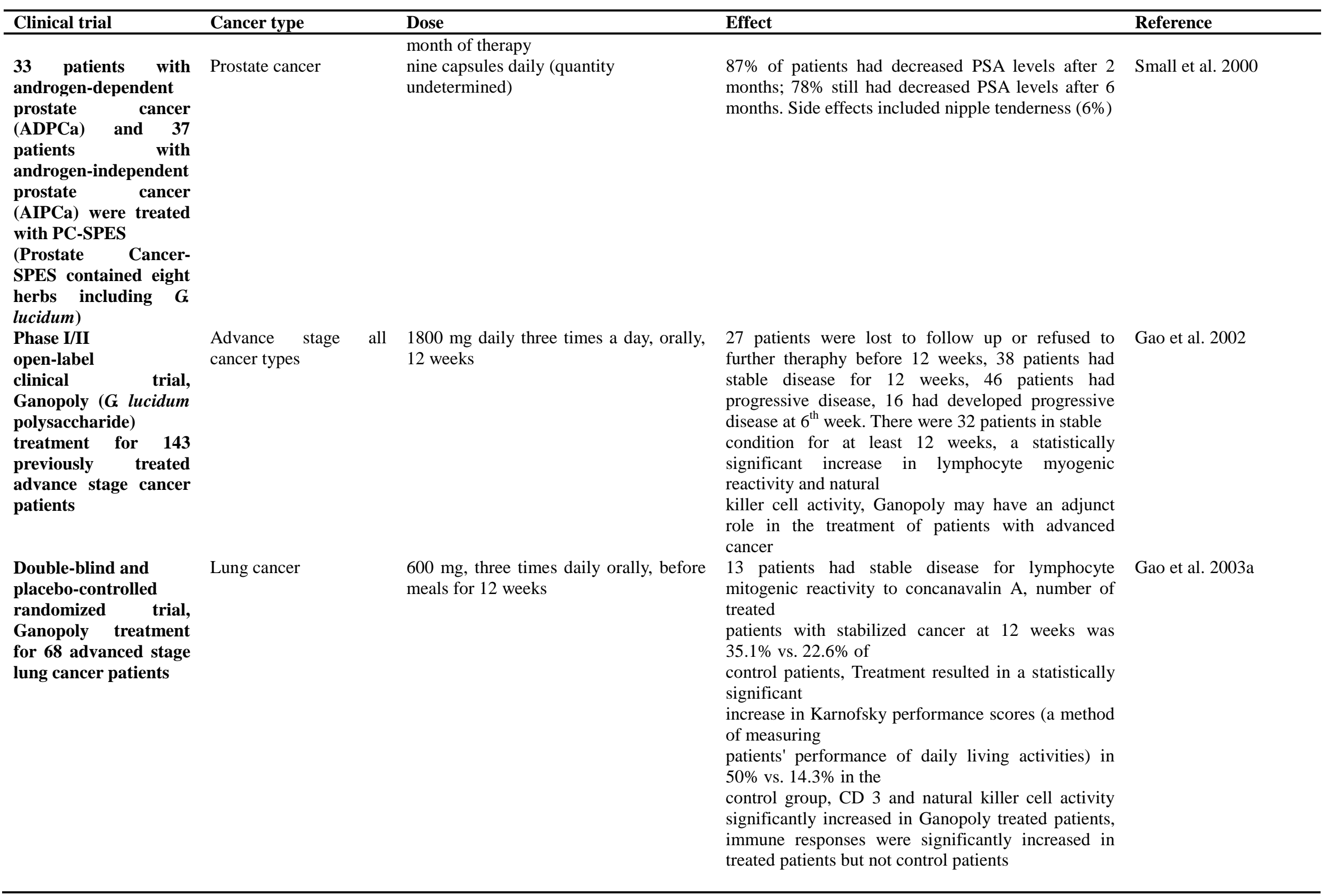




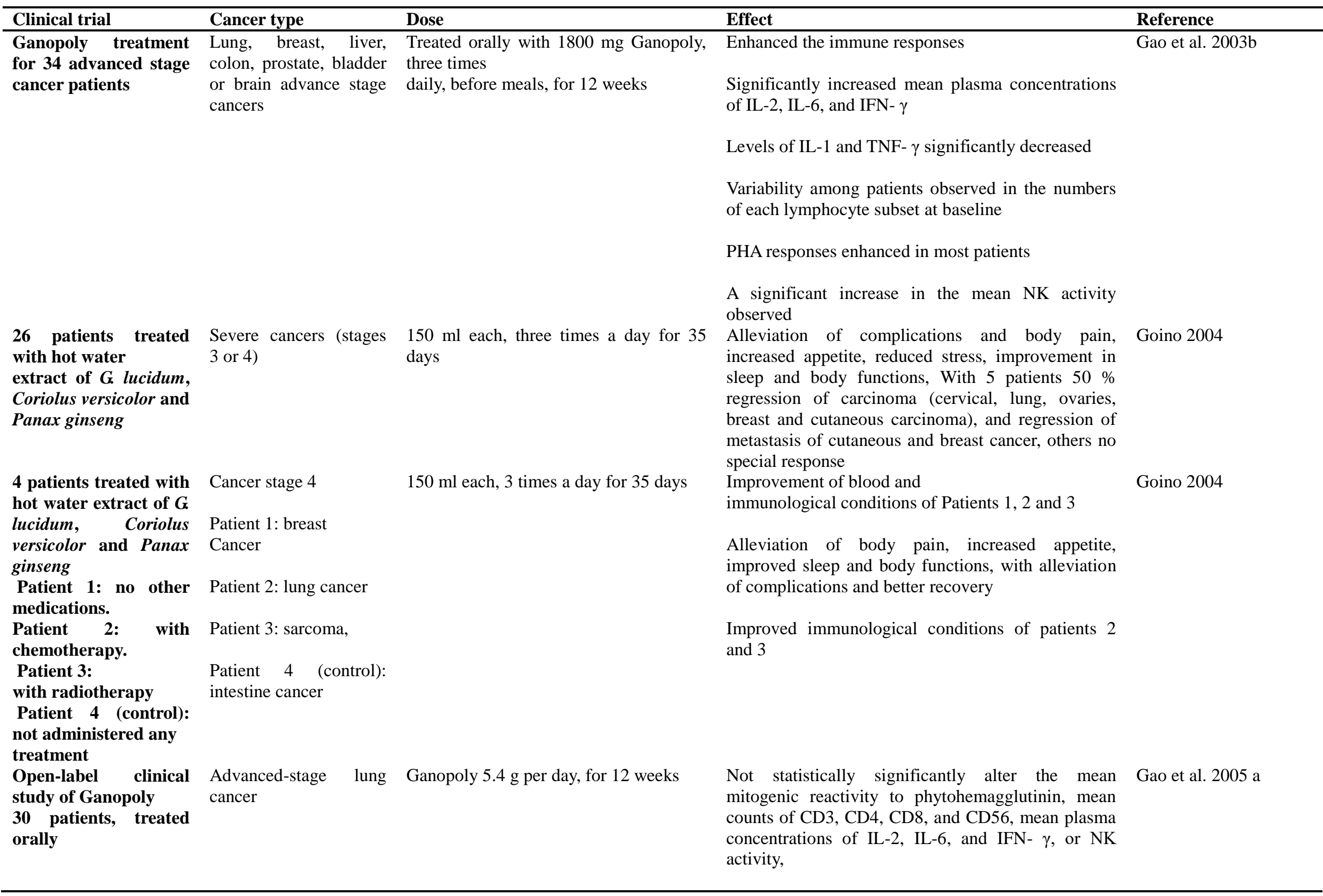




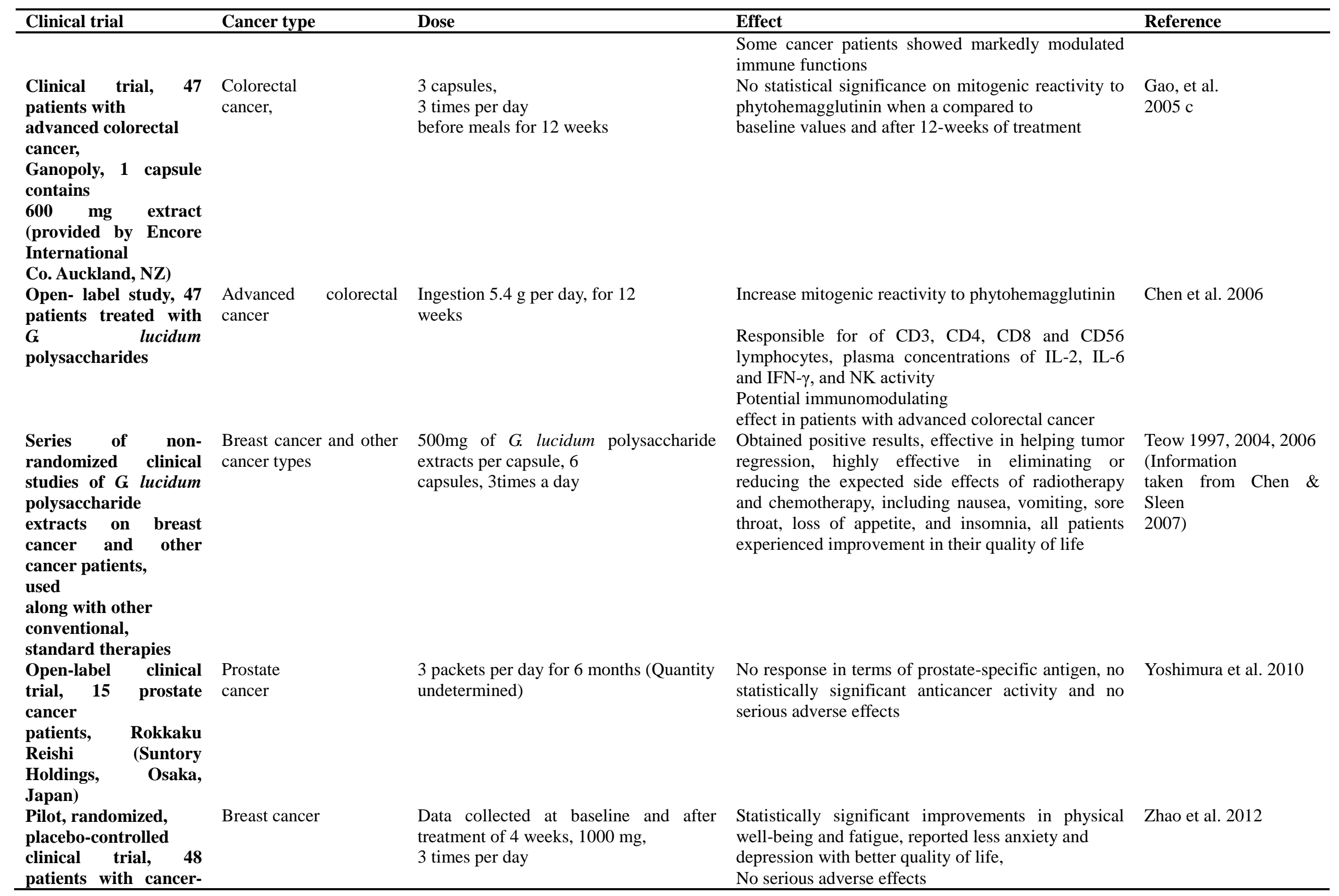




\begin{tabular}{|c|c|c|c|c|}
\hline Clinical trial & Cancer type & Dose & Effect & Reference \\
\hline $\begin{array}{l}\text { Ganopoly TM (crude } \\
\text { polysaccharide } \\
\text { fractions extracted } \\
\text { from } G \text { lucidum, } 143 \\
\text { patients }(83 \text { men and } \\
60 \text { women) having } \\
\text { advanced previously } \\
\text { treated cancer, median } \\
\text { age of } 61 \text { years }\end{array}$ & $\begin{array}{l}\text { Advance tumor (stage } \\
\text { III and IV) }\end{array}$ & $\begin{array}{l}600 \mathrm{mg} \text {, three times daily orally, before } \\
\text { meals for } 12 \text { weeks }\end{array}$ & $\begin{array}{l}\text { Two patients withdrew due to gastrointestinal } \\
\text { toxicity, and } 3 \text { patients died of progressive disease, } \\
27 \text { patients were not assessable for responses and } \\
\text { toxicity, } 11 \text { patients with stable disease at } 6 \text { weeks } \\
\text { and after lost to follow up, Of the } 100 \text { fully } \\
\text { assessable patients, } 46 \text { patients had progressive } \\
\text { disease before or at the } 6 \text { week, } 16 \text { patients } \\
\text { developed progressive disease between } 6 \text { and } 12 \\
\text { weeks of therapy, No complete or partial response } \\
\text { observed, but } 38 \text { of } 143 \text { patients had stable disease } \\
\text { for } 12 \text { weeks or more, prostate-specific antigen } \\
\text { levels in the } 5 \text { prostate cancer patients reduced } \\
\text { significantly during stable disease }\end{array}$ & Zhou et al. 2014 \\
\hline $\begin{array}{l}\text { Randomized double } \\
\text { blind placebo control, } \\
42 \text { patients treated } \\
\text { with water extract and } \\
\text { spores preparation } \\
\text { with a placebo of high } \\
\text { dose vitamin C }\end{array}$ & Gynecological cancer & $\begin{array}{l}\text { Water extract } 1,000 \mathrm{mg} \text { per pack, } G \\
\text { lucidum spores preparation } 1,000 \mathrm{mg} \text { per } \\
\text { pack and vitamin C } 200 \mathrm{mg} \text { per pack, } \\
\text { ingest one package with } 200 \mathrm{ml} \text { of } \\
\text { distilled water one hour before a meal } \\
\text { twice a day for day } 1 \text { and } 2 \text {, increase the } \\
\text { dosage to two packages of drug ingested } \\
\text { with } 200 \mathrm{ml} \text { of distilled water before } \\
\text { meals two times a day on day } 3 \text { and } 4 \text {, } \\
\text { after dosage increased to } 3 \text { packages for } \\
12 \text { weeks }\end{array}$ & $\begin{array}{l}2 \text { patients in the water extract Lingzhi group and } 3 \\
\text { patients in the spore lingzhi group achieved stability } \\
\text { in the disease, all patients in placebo group showed } \\
\text { progression in the disease }\end{array}$ & Suprasert et al. 2014 \\
\hline $\begin{array}{l}\text { Randomized double } \\
\text { blind placebo control, } \\
60 \text { patients who failed } \\
\text { chemotherapy were } \\
\text { treated with } G . \\
\text { lucidum } \\
\text { preparation }\end{array}$ & & & $\begin{array}{l}11,8 \text { and } 5 \text { patients evaluated in water extract, spore } \\
\text { and placebo arms respectively, stable disease that } \\
\text { achieved } 38.1 \% \text { in the water extract arm, } 50 \% \text { in the } \\
\text { spore arm and none in the placebo arm, after one- } \\
\text { year overall survival was } 63.6 \% \text { in the water extract } \\
\text { arm, } 60 \% \text { in the spore arm and } 44 \% \text { in the placebo } \\
\text { arm, slightly improve the immune system with } \\
\text { minimal side effects }\end{array}$ & Suprasert et al. 2015 \\
\hline
\end{tabular}




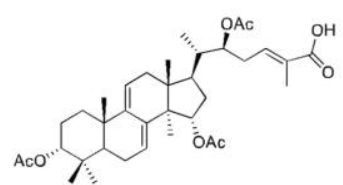

Ganoderic Acid T

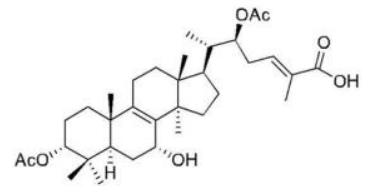

$3 \alpha, 22 \beta$-diacetoxy-7 $\alpha$-hydroxy-5 $\alpha$-lanosta- 8 24 E- dien-26-oic acid

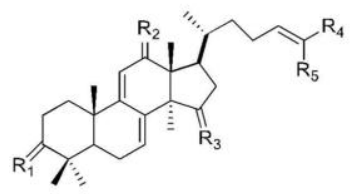

$3 \alpha, 12,15 \beta$-triacetoxy-5a-lanosta-7, 9(11), 24-trien-26-oic acid R1, R3, $\alpha$-OAc, R2, $\alpha$ - $\beta$ $\mathrm{OAc}, \mathrm{R} 4, \mathrm{COOH}, \mathrm{R} 5$
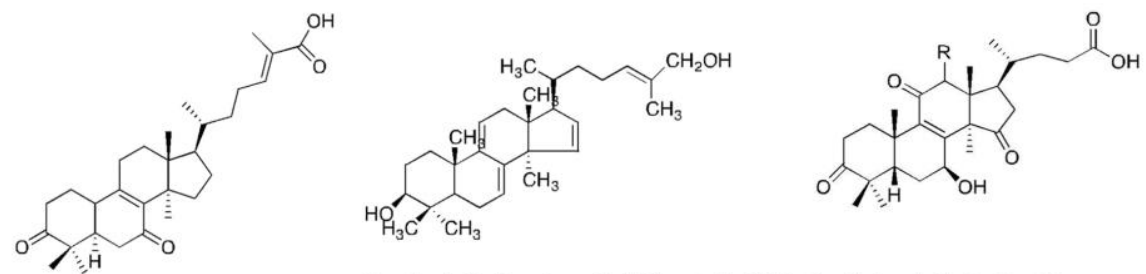

Ganoderol B, Ganodermadiol-5-lanosta-7, 9(11), Lucidenic Acid A, R = H

24-triene-3, 26-diol

Ganoderic Acid DM

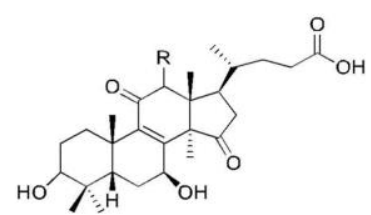

ucidenic Bcid B, $\mathrm{R}=\beta=\mathrm{OH}$

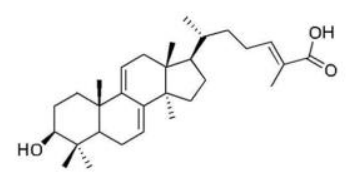

Ganoderic Acid

Lucidenic Acid $C, R=\beta=\mathrm{OH}$

Lucidenic Acid N, R=H

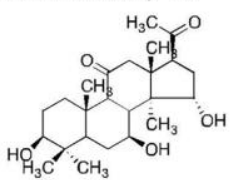

Lucidone C, 3, 7 -dihydroxy-4, 4, 14 trimethyl- 11, 15, 20- trioxo-5-pregn-8-ene

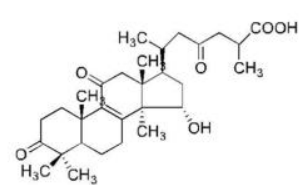

Ganolucidic Acid A, 15 $\alpha$-hydroxy-3, 1 trioxo-5 lanost-8-en-26-oic acid
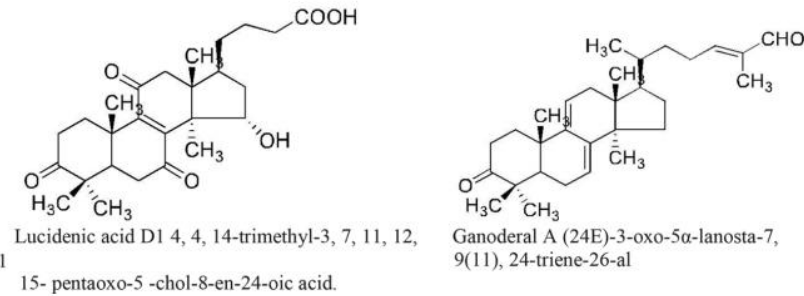

9(11), 24-triene-26-al

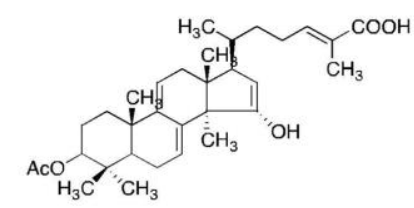

Ganodermic acid R (24E)-3, 15 -diacetoxyepoxy-3, 11, 23-trioxo-5-lanosta-9(11), 20-

5-lanosta- 7, 9(11), 24-triene-26-oic acid

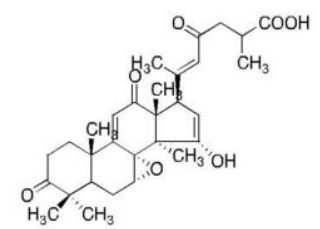

Applanoxidic acid A (20E)-15 hydroxy-7, 8 .

dien-26-oic acid

Fig. 1 - Chemical structures of selected triterpenoids with anti-cancer activities from Ganoderma lucidum (ChemOffice Ultra 2000, Strack 2001 )

\section{Polysaccharides}

Several studies have revealed the medicinal potentials of crude polysaccharides isolated from Ganoderma lucidum (Gao et al. 2003, Shao et al. 2004, Stanley et al. 2005, Kuo et al. 2006). Hung et al. (2008) reported that G. lucidum polysaccharides are believed to initiate an indirect anti-tumor mechanism in the host immune system. Polysaccharides isolated from fruiting bodies contain $(1 \rightarrow 3)$ and/or $\beta$ - $(1 \rightarrow 6)$-D-glucans as main active ingredients and the latter are responsible for immune-modulating and anti-cancer properties (Jones 1992). The anti-cancer effect of polysaccharides arises from enhancement of the host"s immune system, rather through direct cytocidal effects (Gao et al. 2000a, 2000b, Lu et al. 2004, Zhu et al. 2007). These intricate sugars stimulate or modulate the immune system by activating immune cells, such as macrophages (Gao et al. 2003, 2005, Shao et al. 2004, Akramiene et al. 2007, Ahmadi \& Riazipour 2007), B cells (Shao et al. 2004, Manassila et al. 2005), natural killer cells (Gao et al. 2005, Ahmadi \& Riazipour, 2007, Akramiene et al. 2007, Huang \& Ning 2010), T cells (Gao et al. 2003, 2005, Manassila et al. 2005, Ahmadi \& Riazipour 2007). Tumorigenesis was also suppressed by G. lucidum polysaccharides. (Xu et al. 2011). A new polysaccharide isolated from Selenium enriched G. lucidum suppressed proliferation of different cancer cell lines in vitro and induced mitochondria mediated apoptosis (Shang et al. 
2009, 2011). Significant antitumor efficacy was observed through cytotoxic effects on tumor cells by $G$. lucidum polysaccharide nanoparticles in combination with G. lucidum polysaccharides (LZP-F3) (Li et al. 2010b) and arsenic trioxide showed synergistic growth inhibition of human urothelial carcinoma cells (Huang et al. 2010). Two water-soluble sulfated and carboxymethylated G. lucidum polysaccharide derivatives (S-GL and CM-GL) induced cell cycle arrest at the G2/M phase and suppressed the growth of sarcoma tumor cells in vitro and in vivo with low toxicity to animals (Wang et al. 2009). Polysaccharides were also reported to increase the immunoglobin levels to produce an elevated response to antigenic substances such as bacteria, viruses and tumor cells and stimulate TNF- $\alpha$ and IL-6 production, activate NF- $\kappa \mathrm{B}$ and increase hepatotoxicity activity (Gao et al. 2003, Manassila et al. 2005, Kuo et al. 2006). Furthermore, the branched chains of beta-glucans act on complement receptor type 3 (CR-3) triggering a series of molecular pathways such as NF- $\kappa \mathrm{B}$, mitogenactivated protein kinase (MAPK) and protein kinase $\mathrm{C}$ (PKC), which in turn, activate the host immune response for immune cell proliferations (Hong 2004).

Beta-glucans also act on dectin-1 receptor and toll-like receptor 2 (TLR-2) (Brown 2006, Gantner 2003). These actions are enhanced maturation of dendritic cells and increases the opsonic and non-opsonic phagocytosis in turn increase the cytokine production and splenic NK-cell cytotoxicity (Chan 2007). A crude extract of the polysaccharide from fruiting bodies has been used to induce cytokines expression via a toll-like receptors- 4 modulated protein kinase signaling pathway (Guo et al. 2009, De Silva et al. 2013). Chemical components of Ganoderma lucidum spores have potential immunomodulatory effects and anti-tumor activities (Guo et al. 2009, De Silva et al. 2013). When compared to unmodified glucans, chemically modified $\alpha$-D-glucan from spores of G. lucidum have been reported to increasing stimulation effects of lymphocyte proliferation and antibody production (Bao et al. 2001a, b, De Silva et al. 2013). Ganoderan is a biopolymer which has also been used as additional therapy in combination with anti-cancer drugs. It was able to increase the effectiveness of cytotoxic drugs and immunomodulators in patient with prostate cancer (Giavasis 2015). Glucose, galactose, mannose, rhamnose and fucose were detected in G. lucidum polysaccharide hydrolysate (Miyazaki \& Nishijima 1981, Aryantha et al. 2002,). Furthermore, Chang \& Lu (2004) separated polysaccharides of G. lucidum and found that polysaccharide groups composed glucose, D-mannose and D-galactose. Monosaccharaides and oligosaccharides such as glucose with small amounts of other sugar residues such as mannose, fucose, xylose and galactose were observed in highly anti-cancerous preparation (Ooi \& Liu 2000). Fucose is reported to play a role in prevention and treating cancers in mammals (Listinsky et al. 2001, Moriwaki \& Miyoshi 2010, Ale et al. 2011, Miyoshi et al. 2012, Liao et al. 2013). Hence, polysaccharides from G. lucidum can be used to produce carbohydrate-based vaccines with therapeutic efficacy for infectious disease and cancer treatment. G. lucidum has been used for its analgesic and muscle relaxing properties since ancient times (Zhang et al. 2011). Matsuzaki et al. (2013) have demonstrated that G. lucidum polysaccharides exhibit an anti-depressant-like effect and reduces anxiety-type behaviour in rats. Table 3 shows the main polysaccharides having anticancer activity.

\section{Ergosterol}

The pro-vitamin D2, ergosterol (ergosta-5, 7, 22-trien-3 $\beta$-ol) is abundant in Ganoderma lucidum (Paterson 2006). Ergosterol in G. lucidum can be converted to vitamin D2 by ultraviolet (UV) irradiation and hence, produces a variety of photo irradiation products. The principal products are provitamin D2, tachysterol, and lumisterol Ergosterol peroxide $(5 \alpha, 8 \alpha-$ epidioxy-22E-ergosta-6, 2 dien-3 $\beta$-ol). The latter is a steroidal derivative of ergosterol and contributes to various biological activities with strong immunomodulatory and anti-tumor activities (Krzyczkowski et al. 2009). Pro-apoptotic activity of ergosterol peroxide and (22E)ergosta-7, 22-dien-5 $\alpha$-hydroxy-3, 6-dione on prostate cancer cells indicate that these compounds can reduce the growth of prostate cancer cells (Russo et al. 2010). 


\section{Proteins}

The fungal immunomodulatory protein, Ling Zhi-8 (LZ-8), has been regarded as one of the most important bioactive substances produced by Ganoderma lucidum. The recombined Ling Zhi-8 (rLZ-8) is known to improve immune modulatory effects on human monocytederived dendritic cells and induces significant activation and maturation of human dendritic cells (Lin et al. 2009). LZ-8 acts as a promising adjuvant that enhances the efficacy of a DNA cancer vaccine and hence, could be used in preventing and treating various cancers. Further, LZ-8 could activate murine macrophages and T lymphocytes, but other Ganoderma polysaccharides only activate the macrophages (Yeh et al. 2010, De Silva et al. 2013).

\section{Discussion}

\section{Are the beneficial anti-cancer properties truly substantiated?}

To date, the majority of research studies performed on the benefits of using Ganoderma lucidum in traditional Chinese medicine, have been restricted to in vitro and animal studies. Despite the fact that many scientists have postulated a positive association between G. lucidum and treatment of many diseases especially cancer, there are still skepticism and bias in connection with the medicinal potential of this fungus. Observational and anecdotal reports, have not as yet been substantiated by well controlled clinical trials or reliable scientific data as very few studies have been conducted with $G$. lucidum in human cancer patients. These in vitro and animal studies need to be applied to human studies and further clinical trials now as the majority of findings does not translate to the human condition. When going from most sophisticated animal studies into humans, candidates drop out at each level of study. However, some persons are unable or do not wish to tolerate the side effects of radiotherapy or chemotherapy. Hence, they need to turn to herbal remedies or dietary supplements. Their effectiveness, potential side effects, and possible interactions with other drugs are often unclear since these products are not studied and regulated in the same way as drugs. For many of these products, scientific studies are now underway. Public database of clinical trials in the US (Home - ClinicalTrials.gov, access in December 08, 2015) lists five studies of G. lucidum and only two were with cancer patients. The first published clinical trial for anti-cancer activity using G. lucidum was that of Morishige (1988) and 140 breast cancer patients with metastasis were treated with $G$. lucidum essence however only six patients showed promising results (Table 5). Liu \& Chung (2001) reported only one patient diagnosed with Hepatoma, was treated with Ganoderma spores, but there was no standard dosage recorded and the treatment was short term (3-4 months) but there was a significant reduction of the size of the tumor (Table 5). There is a lack of information regarding long term treatment, standard dosage, number of patients treated, placebo control, age, gender and the side effects. Small et al. (2000) recorded a clinical trial (Table 5) treated with PC-SPES), a herbal combination remedy, and a Chinese herbal supplement which contains eight herbs including G. lucidum. It was manufactured by Botanic Lab of Brea, California, USA and introduced to men with prostate cancer in 1996 and decreased PSA levels of cancer. In 2002, this herbal medicine was recalled due to various side effects, such as loss of sex drive, difficulty with erectile function, breast enlargement and tenderness and reduction in body hair since PC-SPES produced an estrogen hormonal effect in the body as PC-SPES samples were found to be contaminated with US Food and Drug Administration (FDA) controlled prescription drugs (White 2002). Gao et al. (2002) has used Ganopoly (G. lucidum polysaccharide extract) treatment for 12 weeks on 143 previously treated advanced stage cancer patients and the results (Table 5) revealed that

Ganopoly was effective for patients, however 46 patients showed progressive disease after $6^{\text {th }}$ week and another 16 patients had developed progressive disease between $6^{\text {th }}$ and $12^{\text {th }}$ week. This research could have been improved by increasing the sample size of the patients, selecting effective dosage, long term treatment and using a standard placebo control. Furthermore, toxicity should be tested since there were side effects such as nausea, insomnia, sweating and diarrhea. Gao et al. (2003a) assessed the effectiveness of Ganopoly treatment on 68 advanced 
stage lung cancer patients in a double-blind and placebo-controlled randomized trial (Table 5). Thirteen patients showed stable disease and lymphocyte mitogenic reactivity to concanavalin A, furthermore, CD 3 percentage and natural killer cell activity significantly increased in Ganopoly treated patients (Gao et al. 2003a). These results demonstrated that Ganopoly can be considered as a supplement for treatment of lung cancer patients. Further Ganopoly treatments with 34 advanced stage cancer patients enhanced immune responses and significantly increased the population of T cells and natural killer cell activity compared to baseline (Table 5, Gao et al. 2003b). Gao et al. (2005) also investigated Ganopoly treatment again as openlabel clinical study for 30 advanced stage lung cancer patients with the same dose as Gao et al. (2003a). Results showed that subgroups of cancer patients are responsive to Ganopoly along with chemotherapy or radiotherapy. Further studies should be continued to determine suitable dosage of the drug, and more trials are needed with a large group of patients, along with radiotherapy and chemotherapy for long term treatments with gender and age base for better results in immune responses. The toxicity of drug should be studied thoroughly since there were side effects such as nausea and insomnia. If Ganopoly treatment is being given along with desired doses of chemotherapy or radiotherapy, then the question arises as to which factor really contributes to positive association with immuno-stimulating effects. Clearly defined protocols, medical standards on exact bioactive compounds and carefully designed experimental controls such as double-blinded, placebo-controlled studies with large trial populations should be performed, so that only Ganopoly variable accounts for better immune responses and not chemotherapy or radiotherapy. Studies on the identification of active ingredients, isolation and purification of individual compounds should be continued and this will enable the active ingredients within Ganopoly to be measured and to understand whether the beneficial compounds in G. lucidum act synergistically or independently.

Hot water extract of three herbs including Ganoderma lucidum was used to treat a few severe stage cancer types (Table 5) along with radiotherapy and chemotherapy under control conditions. The results revealed that it enhanced the blood and immunological conditions of cancer patients (Goino 2004). G. lucidum polysaccharides were taken orally by advanced colorectal cancer patients (Table 5) and results suggested that they have a potential immunomodulating effect in patients (Chen et al. 2006). Teow $(1997,2004,2006)$ performed a series of clinical trials on breast cancer patients using G. lucidum polysaccharide capsules and obtained positive results (Chen \& Sleen 2007). Yoshimura et al. (2010) used Reishi powder packets and performed open label clinical trial for 15 breast cancer patients and obtained no statistically significant anti-cancer activity. Zhao et al. (2012) treated 48 breast cancer patients with a spore powder of G. lucidum (Table 5). Ganopoly TM was applied to advanced tumor (stage III and IV) patients by Zhou et al. (2014) (Table 5). This medical investigation was particularly standard since there was information on age, gender, dosage and adequate patient sample size and most of the patients were not assessable for the treatments. However most of the clinical trials lack information regarding the progress of long term treatments (Goino 2004, Chen et al. 2006, Teow 1997, 2004, 2006, Yoshimura et al. 2010, Zhao et al. 2012, Zhou et al. 2014), have small sample sizes (Goino 2004, Yoshimura et al. 2010, Zhao et al. 2012, Zhou et al. 2014), age (Goino 2004, Teow 1997, 2004, 2006, Yoshimura et al. 2010, Zhao et al. 2012) and placebo control (Teow 1997, 2004, 2006). Suprasert et al. (2014, 2015) carried out successful clinical trials using G. lucidum water extracts and G. lucidum spores for gynecologic cancer patients who failed chemotherapy (Table 5). After long term treatment with Lingzhi, $60 \%$ of the patients showed progressive disease and $40 \%$ showed stable disease (Suprasert et al. 2014). The survival rate was nearly $60 \%$ for both water extract and spores after 1 year of continuous treatment (Suprasert et al. 2015). The results revealed that the drug controlled the disease and slightly improved the immune system with minimal side effects. But it is unclear whether the patientes survival depends on less advance stage of the disease or due to result of treatments. Ganoderma lucidum has a long history as a medicinal mushroom but there is a lack of information corroborating the traditional uses. Hence, to reach its economic and medicinal potential a number of challenges need to be addressed. 


\section{Conclusion}

Accurate scientific evidence is needed for establish the safe and efficient use of Ganoderma lucidum as an integrative therapy for cancers. Experimental, epidemiological, and clinical studies should identify the molecular targets of the cancers and resolve the association between $G$. lucidum intake and cancer risk. Furthermore, the efficacy dosage, efficacy of the drug, and safety, alone or in combination with chemotherapy or radiotherapy should be researched. Investigations should be carried out to determine which pharmacologically active constituents from $G$. lucidum contribute to positive immune responses and exploit the mechanisms of action of G. lucidum at the genetic level or molecular level and with target organs, to fully comprehend their mode of action in cancer treatments. Furthermore, a wider array of toxicity tests should be performed to weigh the pros and cons of cancer treatment among patients and investigate whether the quality of life is improved. More rigorous, reproducible and well-designed scientific studies with adequate sample size and reliable statistical data are also needed to support the associations.

\section{Acknowledgements}

This work was financed by the Science Research Foundation of Guizhou University (No. 201309), the Modernization of Traditional Chinese Medicine Program of Guizhou Province (No. [2012]5008) and Thailand Research Fund Grant - Taxonomy, Phylogeny and Biochemistry of Thai Basidiomycetes (BRG 5580009). Kalani K. Hapuarachchi is grateful to Asanka R. Bandara, Rekhani H. Perera and Yong-Zhong Lu for their valuable suggestions and help.

\section{References}

Ahmadi K, Riazipour M. 2007 - Effect of Ganoderma lucidum on Cytokine Release by Peritoneal Macrophages. Iran. J. Immunol 4, 4, 220-226.

Ahmadi RS, Fasihi RM, Ahmadi K. 2014 - Ganoderma lucidum: A promising antiinflammatory medicinal plant. J Herb Med Pharmacol 3, 1, 67-68.

Akikuni Y. 2003 - Therapeutic agent for a cancer and method of screening the same, and health-care auxiliary food. US20030064076.

Akramiene D, Kondrotas A, Didziapetriene J, Kevelaitis E. 2007 - Effects of beta-glucans on the immune system. Medicina (Kaunas) 43, 597-606.

Ale MT, Maruyama H, Tamauchi H, Mikkelsen JD, Meyer AS. 2011 - Fucoidan from Sargassum sp. and Fucus vesiculosus reduces cell viability of lung carcinoma and melanoma cells in vitro and activates natural killer cells in mice in vivo. Intl. J. Biol. Macromol 49, 331-336.

Anand P, Kunnumakara AB, Sundaram C, Harikumar KB, Tharakan ST, Lai OS, Sung B, Aggarwal BB. 2008 - Cancer is a preventable disease that requires major lifestyle changes. Pharm Res 25, 2097-2116.

Aryantha INP, Adinda A, Kusmaningati S. 2002 - Occurrence of triterpenoids and polysaccharides on Ganoderma tropicum with Ganoderma lucidum as reference. Aust. Mycol 20, 3, 123-129.

Baby S, Johnson AJ, Govindan B. 2015 - Secondary metabolites from Ganoderma. Phytochemistry 114, 66-101.

Bao XF, Duan JY, Fang XY, Fang JN. 2001a - Chemical modifications of the (1, 3)-alpha-Dglucan from spores of Ganoderma lucidum and investigation of their physicochemical properties and immunological activity. Carbohydr. Res 336, 127-140.

Bishop KS, Kao CHJ, Xu Y, Glucina MP, Paterson RRM, Ferguson LR. 2015 - From 2000 years of Ganoderma lucidum to recent developments in nutraceuticals Phytochemistry 114, 56-65.

Boh B, Berovic M, Zhang J, Zhi-Bin L. 2007 - Ganoderma lucidum and its pharmaceutically active compounds. Biotechnol. Annu. Rev 13, 265-301.

Boh B. 2013 - Ganoderma lucidum: A Potential for Biotechnological Production of AntiCancer and Immunomodulatory Drugs. Recent Pat. on Anti-Cancer Drug Discov 8, 
$255-287$.

Borchers AT, Keen CL, Gershwin ME, 2004 - Mushrooms, tumors, and immunity: an update. Exp Biol Med (Maywood) 229, 393-406.

Brown DG. 2006 - Dectin-1: a signaling non-TLR pattern-recognition receptor. Nat. Rev. Immuno 6, 33-43.

Cao QZ, Lin ZB. 2006 - Ganoderma lucidum polysaccharides peptide inhibits the growth of vascular endothelial cell and the induction of VEGF in human lung cancer cell. Life Sci 78, 1457-63.

Cao Y, Wu SH, Dai YC. 2012 - Species clarification of the prize medicinal Ganoderma mushroom "Lingzhi”. Fungal Divers 56, 49-62.

Cao Y, Yuan HS. 2013 - Ganoderma mutabile sp. nov. from Southwestern China based on morphological and molecular data. Mycol Progress 12, 121-126

Chan WK, Law HK, Lin ZB, Lau YL, Chan GC. 2007 - Response of human dendritic cells to different immunomodulatory polysaccharides derived from mushroom and barley. Int. Immuno 19, 7, 891-899.

Chang ST, Buswell JA. 1999 - Ganoderma lucidum (Curt. Fr.) P. Karst. (Aphyllophoromycetideae) - A mushrooming medicinal mushroom. Int J Med Mush 1, 139 - 146.

Chang ST, Mushroom research and development 1996 - equality and mutual benefit. In :( Royse, DJ Penn. ED.) Mushroom Biology and mushroom products. State university, University Park 1-10.

Chang UM, Li CH, Lin LI, Huang CP, Kan LS, Lin SB. 2006 - Ganoderiol F, a Ganoderma triterpene, induces senescence in hepatoma Hep G2 cells. Life Sci 79, 1129-1139.

Chang YW, Lu TJ. 2004 - Molecular characterization of polysaccharides in hot-water extracts of Ganoderma lucidum fruiting bodies. J Food Drug Anal 12, 59-67.

Chen AW, Seleen J. 2007 - Potential benefits of Ling-Zhi or Reishi mushroom Ganoderma lucidum (W. curt. Fr.) P. Karst. (Aphyllophoromycetideae) to breast cancer patients. Int J Med Mushrooms 9, 29-38.

Chen DH, Chen WKD. 2003 - Determination of Ganoderic acids in triterpenoid constituents of Ganoderma tsugae. J Food Drug Anal 11,195-201.

Chen NH, Liu JW, Zhong JJ. 2008 - Ganoderic acid Me inhibits tumor invasion through down-regulating matrix metalloproteinases 2/9 gene expression. J Pharmacol Sci 108, 212-216.

Cheng CR, Yue QX, Wu ZY, Song XY, Tao SJ, Wu XH, Xu PP, Liu X, Guan SH, Guo DA. 2010 - Cytotoxic triterpenoids from Ganoderma lucidum. Phytochemistry 71, 15791585.

Cheng KC, Huang HC, Chen JH, Hsu JW, Cheng HC, Ou CH. 2007 - Ganoderma lucidum polysaccharides in human monocytic leukemia cells from gene expression to network construction. BMC Genomics 8, 411.

Cheng S, Sliva D. 2015 - Ganoderma lucidum for Cancer Treatment: We Are Close but Still Not There, Integr. Cancer Ther 1-9.

Chin SK, Law CL, Cheng PG. 2011 - Effect of drying on crude Ganoderic acids and water soluble polysaccharides content in Ganoderma lucidum. Int J Pharm Sci 3, 38-43.

Chu CL, Chen TC. 2009 - Methods for enhancing innate and adaptive immunity and antigen immunogenicity. US20090285789.

Chung CK, Tong SK. 2003 - Ganoderma lucidum spores for treatment of autoimmune diseases. US20030143246.

Curtis W. 1781 - Flora Londinensis: or plates and descriptions of such plants as grow wild in the environs of London. London: printed by the author. 530.

Dai YC, Cui BK, Yuan HS, Li BD. 2007. - Pathogenic wood-decaying fungi in China. For. Patho 37, 105-120.

Dai YC, Yang ZL. Cui BK, Yu CJ, Zhou LW. 2009 - Species diversity and utilization of medicinal mushrooms and fungi in China. Int J Med Mushr 11, 287-302.

De Silva DD, Rapior S, Fons F, Bahkali AH, Hyde KD. 2012a - Medicinal mushrooms in supportive cancer therapies: an approach to anti-cancer effects and putative mechanisms of action. Fungal Divers 55, 1-35.

De Silva DD, Rapior S, Sudarman E, Stadler M, Xu J, Alias SA, Hyde KD. 2013 - Bioactive metabolites from macrofungi: ethnopharmacology, biological activities and chemistry. Fungal Divers 62, 1-40. 
Dong C, Han Q. 2015 - Ganoderma lucidum (Lingzhi, Ganoderma): Fungi, algae, and other materials In: Liu Y, Wang Z, Zhang J. (Eds) Dietary Chinese Herbs Chemistry: Pharmacology and Clinical Evidence Springer, London 759-765.

Fries EM. 1821 - Systema Mycologicum, sistens fungorum ordines, genera etspecies. 1. Gryphiswaldiae: Sumtibus Ernesti Mauritti. 353.

Gantner BN, Simmons RM, Canavera SJ, Akira S, Underhill DM. 2003 - Collaborative induction of inflammatory responses by dectin-1 and toll-like receptor 2. J Exp. Med 197, 9, 1107-17.

Gao JJ, Min BS, Ahn E, Nakamura N, Lee HK. 2002 - New triterpene aldehydes, lucialdehydes A-C, from Ganoderma lucidum and their cytotoxicity against murine and human tumor cells. Chem Pharm Bull 50, 837-840.

Gao XX, Wang BX, Fei XF, Zhang J, Gong YJ, Minami M, Nagata T, Ikejima T. 2000b Effects of polysaccharides (FI0-crom mycelium of Ganoderma tsugae on proinflammatory cytokine production by THP-1 cells and human PBMC (II). Acta Pharmacol. Sin 21, 1186-1192.

Gao XX, Fei XF, Wang BX, Zhang J, Gong YJ, Minami M, Nagata T, Ikejima T. 2000a Effects of polysaccharides (FI0-b) from mycelium of Ganoderma tsugae on proinflammatory cytokine production by THP-1 cells and human PBMC (I). Acta Pharmacol. Sin 21, 1179- 1185.

Gao Y, Gao H, Chan E, editors. 2005 - Antitumor activity and underlying mechanisms of Ganopoly, the refined polysaccharides extracted from Ganoderma lucidum, in mice. Immunol Invest 34, 171-98.

Gao YH, Dai XH, Chen G, Ye J-X, Zhou SF. 2003a - A Randomized, Placebo-Controlled, Multicenter Study of Ganoderma lucidum (W.Curt:Fr.) Lloyd (Aphyllophoromycetideae) Polysaccharides (Ganopoly ${ }^{\circledR}$ ) in Patients with Advanced Lung Cancer. Int. J. of Med. Mushrooms 5, 4.

Gao YH, Zhou SF, Jiang WQ, Huang M, Dai XH. 2003b - Effects of Ganopoly (a Ganoderma lucidum polysaccharide extract) on immune functions in advanced-stage cancer patients. Immunol Invest 32, 201-15.

Giavasis I. 2015 - Polysaccharides Natural Fibers in Food and Nutrition, Edition: 1st, Chapter: 8, Publisher: CRC Press 2014, Editors: Noureddine Benkeblia 171-206. doi: $10.1201 / \mathrm{b} 17121-9$ in book.

Goino T. 2004 - Physiologically active compositions based upon active ingredients of basidiomycotina and araliaceae. US 6746675.

Grienke I, Kaserer T, Pfluger F, Mair CE, Langer T, Schuster D, Rollinge JM. 2004 Accessing biological actions of Ganoderma secondary metabolites by in silico profiling. Phytochemistry $114-124$.

Guo L, Xie J, Ruan Y, Zhou L, Zhu H, Yun X. 2009 - Characterization and immuno stimulatory activity of a polysaccharide from the spores of Ganoderma lucidum. Int. Imm. pharmaco 9, 1175-1182.

Hapuarachchi KK, Wen TC, Deng CY, Kang JC, Hyde KD - Mycosphere Essays 1: Taxonomic confusion in the Ganoderma lucidum species complex. Mycosphere 6, 5, 542-559, Doi 10.5943/mycosphere/6/5/4.

Hawksworth DL. 2005 - Reflections on changing names and related nomenclatural issues in edible and medicinal mushrooms. Int J Med Mushrooms 7, 29-38.

Hong F, Yan J, Baran JT, Allendorf DJ, Hansen RD, Ostroff GR. 2004 - Mechanism by which orally administered beta-1-3-glucans enhance the tumoricidal activity of antitumor monoclonal antibodies in murine tumour models. The J. of Immuno 173, 2, 797-806.

Hossain A, Radwan FF, Doonan BP. 2012 - A possible cross-talk between autophagy and apoptosis in generating an immune response in melanoma. Apoptosis 17, 10, 10661078 https://clinicaltrials.gov/.

Hu H, Ahn NS, Yang X, Lee YS, Kang KS. 2002 - Ganoderma lucidum extract induces cell cycle arrest and apoptosis in MCF-7 human breast cancer cell. Int J Cancer 102, 250253.

Huang S-Q, Ning Z-X. 2010 - Extraction of polysaccharide from Ganoderma lucidum and its immune enhancement activity. Int. J. of Bio. Macromol 47 336-341.

Huang WT, Wang SH, Chen CH, Yang WB. 2008 - Structure determination of $\beta$ glucans from 
Ganoderma lucidum with matrix assisted laser desorption/Ionization (MALDI) mass spectroscopy. Molecules 13, 8, 1538-1550.

Jang KJ, Han MH, Lee BH, Kim BW, Kim CH, Yoon HM, Choi YH. 2010 - Induction of apoptosis by ethanol extracts of Ganoderma lucidum in human gastric carcinoma cells. J Acupunct Meridian Stud 3, 1, 24-31.

Jedinak A, Thyagarajan-Sahu A, Jiang J, Sliva D. 2011 - Ganodermanontriol, a lanostanoid triterpene from Ganoderma lucidum, suppresses growth of colon cancer cells through ss-catenin signaling. Int J Oncol 38-767.

Jiang J, Grieb B, Thyagarajan A, Sliva D. 2008 - Ganoderic acids suppress growth and invasive behaviour of breast cancer cells by modulating AP-1 and NF-kappa B signaling. Int J Mol Med 21, 5, 577-584.

Jiang J, Jedinak A, Sliva D. 2011 - Ganodermanontriol (GDNT) exerts its effect on growth and invasiveness of breast cancer cells through the down-regulation of CDC20 and uPA. Biochem Biophys Res Commun. 415, 2, 325-329.

Jiang J, Slivova V, Valachovicova T, Harvey K, Sliva D. 2004 - Ganoderma lucidum inhibits proliferation and induces apoptosis in human prostate cancer cells PC-3. Int J Oncol 24, 109.

Jin X, Beguerie JR, Sze DM, Chan GCF. 2012 - Ganoderma lucidum (Reishi mushroom) for cancer treatment. Cochrane Database of Sys Rev 6. Art. No. CD007731.

Jin X, Beguerie JR, Sze DM, Chan GCF. 2016 - Ganoderma lucidum (Reishi mushroom) for cancer treatment. Cochrane Database of Sys Rev 4. Art. No. CD007731. doi: 10.1002/14651858.CD007731.pub3.

Jones K. 1992 - Reishi: Ancient Herb for Modern Times. Sylvan Press, Seattle, WA, 48 Krzyczkowski W, Malinowska E, Suchocki P, Kleps J, Olejnik M, Herold F. 2009 Isolation and quantitative determination of ergosterol peroxide in various edible mushroom species. Food Chem 351-355.

Kuo MC, Weng CY, Ha CL, Wu MJ. 2006 - Ganoderma lucidum mycelia enhance innate immunity by activating NF-kappa. J Ethnopharmacol. 103, 217-22.

Lai T, Gao Y, Zhou SF. 2004 - Global marketing of medicinal Ling Zhi mushroom Ganoderma lucidum (W.Curt:Fr.) Lloyd (Aphyllophoromycetideae) products and safety concerns. Int J Med Mushr 6, 189-194.

Li F, Wang Y, Wang X, Li J, Cui H, Niu M. 2012 - Ganoderic acids suppress growth and angiogenesis by modulating the NF-kappa signaling pathway in breast cancer cells. Int J Clin Pharmacol Ther 50, 10, 712-721.

Li J, Zhang J, Chen H, Chen X, Lan J, Liu C 2013 - Complete Mitochondrial Genome of the Medicinal Mushroom Ganoderma lucidum. PLoS ONE 8, 8 e72038. doi:10.1371/journal.pone.0072038.

Li N, Hu YL, He CX, Hu CJ, Zhou J, Tang GP, Gao JQ. 2010b - Preparation, characterization, and anti-tumour activity of Ganoderma lucidum polysaccharide nanoparticles. J. of Phar. and Pharmaco 62, 1, 139-144.

Li YB, Liu RM, Zhong JJ. 2013 - A new Ganoderic acid from Ganoderma lucidum mycelia and its stability. Fitoterapia. 84, 115-122.

Liao SF, Liang CH, Ho MY, Hsu TL, Tsai TI, Hsieh YSY, Tsai CM, Lib ST, Cheng YY, Tsaoe SM, Lin TY, Linc ZY, Yang WB, Ren CT, Lin KI, Khooc KH, Linc CH Hsub HY, Wub CY, Wong CH. 2013a - Immunization of fucose-containing polysaccharides from Reishi mushroom induces antibodies to tumor-associated Globo H-series epitopes. PNAS 110, 34, 13809-13814.

Lin CN, Tome WP, Won SJ. 1991 - Novel cytotoxic principles of Formosan Ganoderma lucidum. J. Nat. Prod 54, 998-1002.

Lin SB, Li CH, Lee SS, Kan LS. 2003 - Triterpene-enriched extracts from Ganoderma lucidum inhibit growth of hepatoma cells via suppressing protein kinase $\mathrm{C}$, activating mitogen-activated protein kinases and G2-phase cell cycle arrest. Life Sci 72, 23812390.

Lin YL, Liang YC, Tseng YS, Huang HY, Chou SY, Hseu RS, Huang CT, Chiang BL. 2009 An immunomodulatory protein, Ling Zhi-8, induced activation and maturation of human monocyte-derived dendritic cells by the NF- $\kappa B$ and MAPK pathways. J. of 
Leuk. Bio 86, 879.

Listinsky JJ, Listinsky CM, Alapati V. 2001 - Cell surface fucose ablation as a therapeutic strategy for malignant neoplasms. Adv. Anat. Pathol 8, 330-337.

Liu B. 1974 - The Chinese medical fungi. Shanxi People ${ }^{e e}$ Press, Taiyuan 1-196 (in Chinese).

Liu J, Shimizu K, Tanaka A, Shinobu W, Ohnuki K, Nakamura T, Kondo R. 2012d - Target proteins of Ganoderic acid DM provides clues to various pharmacological mechanisms. Sci Rep 2, 905. doi: 10.1038/srep00905.

Liu RM, Li YB, Zhong JJ. 2012c - Cytotoxic and pro-apoptotic effects of novel Ganoderic acid derivatives on human cervical cancer cells in vitro. Eur J Pharmacol 681(1-3), $23-33$.

Liu RM, Zhong JJ. 2011 - Ganoderic acid Mf and S induce mitochondria mediated apoptosis in human cervical carcinoma HeLa cells. Phytomed 18, 5, 349-355.

Liu X, Chung C.K. 2001 - Germination-activated red Ganoderma lucidum spores and method for producing the same. EP1092765.

Liu X, Yuan, Chung CK, Chen XJ. 2002 - Anti-tumor activity of the sporoderm broken germinating spores of Ganoderma lucidum. Cancer Lett 182, 155-161.

Liu YH, Lin YS, Lin KL, Lu YL, Chen CH, Chien MY, Shang HF, Lin SY, Hou WC. 2015b Effects of hot-water extracts from Ganoderma lucidum residues and solid-state fermentation residues on prebiotic and immune-stimulatory activities in vitro and the powdered residues used as broiler feed additives in vivo. Bot. Studies, An Int 56, 17.

Lu Q. Y, Jin Y. S, Zhang Q, editors. 2004 - Ganoderma lucidum extracts inhibit growth and induce actin polymerization in bladder cancer cells in vitro. Cancer Lett. 216, 9-20.

Manassila M, Sooksa-Nguan T, Boonkerd N, Rodtong S, Teaumroong N. 2005 - Phylogenetic diversity of wild edible Russula from northeastern Thailand on the basis of internal transcribed spacer sequence. Science Asia 31, 323-328.

Mao XL. 1998 - Economic fungi of China. Science Press, Beijing 1-762 (in Chinese).

Matsuzaki H, Shimizu Y, Iwata N, Kamiuchi S, Suzuki F, lizuka H, Hibino Y, Min BS, Gao JJ, Nakamura N, Hattori M. 2000 - triterpenes from the spores of Ganoderma lucidum and their cytotoxicity against meth-A and LLC tumor cells. Chem Pharm Bull 48, $1026-1033$.

Min BS, Gao JJ, Nakamura N, Hattori M. 2000 - Triterpenes from the spores of Ganoderma lucidum and their cytotoxicity against Meth-A and LLC tumor cells. Chem. Pharm. Bull 48, 1026-1033.

Miyazaki T, Nishijima M. 1981 - Studies on fungal polysaccharides. XXVII. Structural examination of a water-soluble, antitumor polysaccharide of Ganoderma lucidum. Chem Pharm Bull 29, 3611-16.

Miyoshi H, Ajima R, Luo CT, Yamaguchi TP, Stappenbeck TS. 2012 - Wnt5a Potentiates TGF- $\beta$ Signaling to Promote Colonic Crypt Regeneration after Tissue Injury. Science. 338, 6103, 108-113.

Moriwaki K, Miyoshi E. 2010 - Fucosylation and gastrointestinal cancer. World J Hepatol 2, 151-161.

NCI. $2011 \quad-\quad$ National cancer institute. Cancer topics http://www.cancer.gov/cancertopics/cancerlibrary/what-is-cancer.

Nguyen PDN, Do HT, Le BD. 2013 - Characteristics of ecological factors and their distribution of Ganodermataceae Donk. in highlands of Vietnam. J. Biol 35, 198-205.

Noguchi M, Kakuma T, Tomiyasu K, Konishi F, Kumamoto S, Kondo R. 2005 - Phase I study of a methanol extract of Ganoderma lucidum, edible and medicinal mushroom, in men with mild symptoms of bladder outlet obstruction. Urology 66 (Suppl 3A), 21.

NonakaY, Ishibashi H, Nakai M, Shibata H, KisoY, Abe S. 2008 - Effects of the antlered form of Ganoderma lucidum on tumor growth and metastasis in cyclophosphamide-treated mice. Biosci Biotechnol Biochem 72, 6, 1399-1408.

Okazaki M. 2013 - Antidepressant-like effects of a water-soluble extract from the culture medium of Ganoderma lucidum mycelia in rats. BMC Complement. Altern. Med 13, 370.

Ooi VE, Liu F. 2000 - Immunomodulation and anti-cancer activity of polysaccharide-protein complexes. Curr Med Chem 7, 715-729.

Pan K, Jiang Q, Liu G, Miao X, Zhong D. 2013 - Optimization extraction of Ganoderma 
lucidum polysaccharides and its immunity and antioxidant activities. Int. J. Biol Macromol 55, 301- 306.

Pang X, Chen Z, Gao X, Liu W, Slavin M, Yao W. 2007 - Potential of a novel polysaccharide preparation (GLPP) from Anhui-grown Ganoderma lucidum in tumor treatment and immunostimulation. J Food Sci 72, S435-S42.

Paterson RRM. 2006 - Ganoderma - a therapeutic fungal bio factory. Photochemistry 67, 1985-2001.

Patouillard NT. 1889 - Le genre Ganoderma. Bull. Soc. Mycol. France 5, 64-80.

Radwan FF, Hossain A, God JM. 2015 - Reduction of myeloid derived suppressor cells and lymphoma growth by a natural triterpenoid. J Cell Biochem 116, 1, 102-114.

Ruan W, Lim AH, Huang LG, Popovich DG. 2014 - Extraction optimization and isolation of triterpenoids from Ganoderma lucidum and their effect on human carcinoma cell growth. Nat Prod Res 28, 24, 2264-2272.

Ruddon RW - 2007 Cancer biology, 4th edn. Oxford University Press US.

Russo A, Cardile V, Piovano M, Caggia S, Espinoza CL, Garbarino JA. 2010 - Pro-apoptic activity of ergosterol peroxide and (22E)-ergosta-7, 22-dien-5a-hydroxy-3, 6-dione in human prostate cancer cells. Chemico-Biolo. Inter 184, 3, 352-358.

Ryvarden L, Johansen I. 1980 - A preliminary polypores flora of East Africa. Fungi flora, Oslo, 1-636.

Shang D, Li Y, Wang C, Wang X, Yu Z, Fu X. 2011 - novel polysaccharide from Se-enriched Ganoderma lucidum induces apoptosis of human breast cancer cells. Reports 25, 267272.

Shang D, Zhang J, Wen L, Li Y, Cui Q. 2009 - Preparation, characterization, and antiproliferative activities of the Se-containing polysaccharide Se GLP-2B-1 from Seenriched Ganoderma lucidum. J. Agric Food Chem 57, 77, 37-42.

Shao BM, Dai H, Xu W, Lin ZB, Gao XM. 2004 - Immune receptors for polysaccharides from Ganoderma lucidum. Biochem. Biophys. Res. Commun 323, 133-141.

Shi KG, Quing LH. 2002 - The follow-up observation assessment of medium and late phases cancer treated by Chinese Ganoderma lucidum essence (CGLE). Ganoderma: Genetics, chemistry, pharmacology and therapeutics, Proceedings of International Symposium on Ganoderma Research. Shanghai, China October 21-23.

Singh S, Harsh NSK, Gupta PK. 2015 - Potential role of host tree species in determining the composition of polysaccharides of Ganoderma lucidum (Fr.) Karst. (GLPS). Current Rese in Environm \& App Myco 5, 3, 196-201.

Singh SK, Doshi A, Pancholy A, Pathak R. 2013 - Biodiversity in wood-decay macro-fungi associated with declining arid zone trees of India as revealed by nuclear rDNA analysis. Eur J Plant Pathol 1-10.

Siwulski M, Sobieralski K, Golak-Siwulska I, Sokol S, Sękara A. 2015 - Ganoderma lucidum (Curt. Fr.) Karst. - health-promoting properties. A review. Herba Pol. 61, 3, 105- 118.

Sliva D, Loganathan J, Jiang J. 2012 - Mushroom Ganoderma lucidum prevents colitisassociated carcinogenesis in mice. PLoS One. 7, 10, e47873.

Sliva D. 2003 - Ganoderma lucidum (Reishi) in cancer treatment. Integr Cancer Ther 2:358364.

Sliva D. 2006 - Ganoderma lucidum in cancer research. Leuk. Res 30, 767-768.

Small EJ, Frohlich MW, Bok R, Shinohara K, Grossfeld G, Rozenblat Z, Kelly WK, Corry M, Reese DM . 2000 - Prospective Trial of the Herbal Supplement PC-SPES in Patients with Progressive Prostate Cancer. J. Clinical Oncology 18, 21, 3595-3603.

Stanley G, Harvey K, Slivova V, Jiang J, Sliva D. 2005 - Ganoderma lucidum suppresses angiogenesis through the inhibition of secretion of VEGF and TGF-betal from prostate cancer cells. Biochem Biophys Res Commun. 330, 46-52.

Strack D. 2001 - ChemOffice Ultra 2000.Phytochemistry 57, 1, 144.

Sun B, Cai YY, Li YS. 2013 - The nonstructural protein NP1 of human Boca virus 1 induces cell cycle arrest and apoptosis in Hela cells. Virolo 440, 75-83.

Sun F, Zhang X. 2011. Recombinant Ganoderma lucidium immunomodulatory protein (rLZ-8) and uses thereof. US2011009597.

Sun LX, Lin ZB, Li XJ, Li M, Lu J, Duan XS, Ge ZH, Song YX, Xing EH, Li WD. 2011 Promoting effects of Ganoderma lucidum polysaccharides on B16F10 cells to activate 
lymphocytes. Basic Clin. Pharmacol. Toxicol 108, 149-154

Suprasert P, Apichartpiyakul C, Sakonwasun C, Nitisuwanraksa P, Phuackchantuck R. 2014 Clinical Characteristics of Gynecologic Cancer Patients who Respond to Salvage Treatment with Lingzhi. Salvage Treatment in Gynecologic Cancer Cases with Lingzhi. Asian Pac J Cancer Pre 5 10, 4193-4196.

Suprasert P, Apichartpiyakul C, Sakonwasun C, Nitisuwanraksa P, Phuackchantuck R. 2015 A Randomized Double Blinded Study of Ganoderma lucidum (Lingzhi) in Salvage Setting of Recurrent Gynecologic Cancer. Int J Cancer Clin Res 2, 021.

Tan WC. 2015 - Ganoderma neo-japonicum Imazeki revisited: Domestication study and antioxidant properties of its basidiocarps and mycelia. Sci. Rep 5, 12515, doi: $10.1038 /$ srep 12515.

Tang W, Gu T, Zhong JJ. 2006a - Separation of targeted Ganoderic acids from Ganoderma lucidum by reversed phase liquid chromatography with ultraviolet and mass spectrometry detections. Biochem Eng J 32, 205-210.

Tang W, Liu JW, Zhao WM, Wei DZ, Zhong JJ. 2006b - Ganoderic acid T from Ganoderma lucidum mycelia induces mitochondria mediated apoptosis in lung cancer cells. Life Sci 80, 205-211.

Tasaka K, Akagi M, Miyoshi K, Mio M, Makino T. 1988a - Anti-allergic constituents in the culture medium of Ganoderma lucidum. (I). Inhibitory effect of oleic acid on histamine release. Agents Actions 23, 153-156.

Tasaka, K., Mio, M., Izushi, K., Akagi, M. and Mkino, T. 1988b - Anti-allergic constituents in the culture medium of G. lucidum. (II). The inhibitory effect of cyclooctasulfur on histamine release. Agents Actions 23, 157-160.

Teng SC. 1934 - Notes on Polyporaceae from China. Sinensia 5, 198-200.

Tomasi S, Lohezic-Le DF, Sauleau P, Bezivin C, Boustie J. 2004 - Cytotoxic activity of methanol extracts from Basidiomycete mushrooms on murine cancer cell lines. Pharmazie. 59, 290-3.

Wang J, Zhang L, Yu Y, Cheung PCK. 2009 - Enhancement of Antitumor Activities in Sulfated and Carboxymethylated Polysaccharides of Ganoderma lucidum J. Agric. Food Chem 2009, 57, 10565-10572.

Wang J, Zhang L. 2009 - Structure and chain conformation of five water soluble derivatives of $\beta$-D-glucan isolated from Ganoderma lucidum. Carbohydr. Res 344, 105-112.

Wang XC, Xi RJ, Li Y, Wang DM, Yao YJ. 2012 - The species identity of the widely cultivated Ganoderma, 'G. lucidum' (Ling-zhi), in China. PLoS ONE 7-40857.

Watanabe K, Shuto T, Sato M, Onuki K, Mizunoe S, Suzuki S, Sato T, Koga T, Suico MA, Kai H, Ikeda T 2011 - Lucidenic acids-rich extract from antlered form of Ganoderma lucidum enhances TNF $\alpha$ induction in THP-1 monocytic cells possibly via its modulation of MAP kinases p38 and JNK. Biochem Biophys Res Commun 408, 1, 18-24.

Weng CJ, Chau CF, Hsieh YS, Yang SF, Yen GC. 2008 - Lucidenic acid inhibits PMAinduced invasion of human hepatoma cells through inactivating MAPK/ERK signal transduction pathway and reducing binding activities of NF-kappa and AP-1. Carcinogen 29, 1:147-156.

Weng CJ, Yen GC. 2010 - The in vitro and in vivo experimental evidences disclose the chemo preventive effects of Ganoderma lucidum on cancer invasion and metastasis. Clin Exp Metastasis 27, 361-369.

WHO. 2011 - World Health Organization. Diabetes program. http://wwwwho.int/mediacentre/factsheets/fs312/en/.

WHO. $2014 \quad-\quad$ World Health Organization. Diabetes program. http://www.who.int/mediacentre/factsheets/fs312/en/.

White J. 2002 - PC-SPES - A Lesson for Future Dietary Supplement Research. Editorials. Journal of the National Cancer Institute 94, 17.

Wong KP, Wong MC. 2003 - Compositions containing an active fraction isolated from Ganoderma lucidum and methods of use. US2003095981.

World cancer Research International ( http://www.wcrf.org/).

Wu GS, Guo JJ, Bao JL, Li XW. 2013 - Anti-cancer properties of triterpenoids isolated from Ganoderma lucidum - a review. Expert Opin. Investig. Drugs 22, 981-992.

Wu SH, Dai YC, Hattori T. 2012 - Species clarification for the medicinally valuable 
sanghuang" mushroom. Bot Stud 53, 135-149.

Xu K, Liang X, Gao F, Zhong J, Liu J. 2010 - Antimetastatic effect of Ganoderic acid T in vitro through inhibition of cancer cell invasion. Process Biochem 45, 1261-1267.

Xu Z, Chen X, Zhong Z, Chen L 2011 - Ganoderma lucidum polysaccharides: immunomodulation and potential anti-tumor activities. Am. J. Chin. Med 39, 15-27.

Yang LZ, Feng B. 2013 - what is the Chinese "Lingzhi"? - A taxonomic mini-review, Mycology, An Int. J. on Fungal Bio 4, 1, 1-4.

Yao X, Li G, Xu H, Lu C. 2012 - Inhibition of the JAK-STAT3 signaling pathway by Ganoderic acid A enhances chemosensitivity of HepG2 cells to cisplatin. Planta Med $78,16,1740-1748$.

Ye L-B, Zheng X, Zhang J, Tang Q, Yang Y, Wang X, Li J, - Y-F, Pan Y-J. 2011 Biochemical characterization of a proteoglycan complex from an edible mushroom Ganoderma lucidum fruiting bodies and its immunoregulatory activity. Food Res Int 44, 367-372.

Yeh CH, Chen HC, Yang JJ, Chuang WI, Sheu F. 2010 - Polysaccharides PS-G and protein LZ-8 from Reishi (Ganoderma lucidum) exhibit diverse functions in regulating murine macrophages and $\mathrm{T}$ lymphocytes. J Agric Food Chem 58, 15, 8535-44. doi: $10.1021 / \mathrm{jf} 100914 \mathrm{~m}$.

Yoshimura K, Kamoto T, Ogawa O, Matsui S, Tsuchiya N, Tada H, Murata K. Yoshimura K, Habuchi T, Fukushima M. 2010 - Medical mushrooms used for biochemical failure after radical treatment for prostate cancer. An open-label study. International Journal of Urology 17, 6, 548-554. doi: 10.1111/j.1442-2042.2010.02528.x.

Ying JZ, Mao ZL, Ma QM, Zong LC, Wen HA. 1987 - Icons of medicinal fungi from China. Science Press, Beijing 1-579 (in Chinese).

Yue QX, Xie FB, Guan SH. 2008 - Interaction of Ganoderma triterpenes with doxorubicin and proteomic characterization of the possible molecular targets of Ganoderma triterpenes. Cancer Sci 99, 7, 1461-1470.

Yuen WMJ, Gohel MDI. 2005 - Anti-cancer Effects of Ganoderma lucidum: A Review of Scientific Evidence, Nutrition and Cancer 53, 1, 11-17.

Yu YN, Shen MZ. 2003 - The history of Lingzhi (Ganoderma spp.) cultivation. Mycosystema. 22, 3-9 (in Chinese).

Zaidman BZ, Yassin M, Mahajna J, Wasser SP. 2005 - Medicinal mushroom modulators of molecular targets as cancer therapeutics. Appl Microbiol Biotechnol 67, 453-468.

Zhang J, Tang Q, Zhou C, Jia W, Da Silva L, Nguyen LD, Reutter W, Fan H. 2010 - GLIS, a bioactive proteoglycan fraction from Ganoderma lucidum, displays anti-tumour activity by increasing both humoral and cellular immune response. Life sciences 87 , 19-22, 628-637.

Zhang XQ, Ip FC, Zhang DM, Chen LX, Zhang W, Li YL, Ip NY, Ye WC. 2011 Triterpenoids with neurotropic activity from Ganoderma lucidum. Nat. Prod. Res 25, 1607-1613.

Zhao H, Zhang QY, Zhao L, Huang X, Wang JC, Kang XM. 2012 - Spore powder of Ganoderma lucidum improves cancer-related fatigue in breast cancer patients undergoing endocrine therapy: a pilot clinical trial. Evid Based Complement Alternat Med Art. No. 809614. doi:10.1155/2012/809614.

Zhou LW, Cao, Y, Wu SH, Vlasák J, Li DW, Li MJ, Dai YC. 2015. - Global diversity of the Ganoderma lucidum complex (Ganodermataceae, Polyporales) inferred from morphology and multilocus phylogeny. Phytochemistry114, 7-15.

Zhou SF, Gao Y, Chen GL, Dai XH, Ye JX. 2014 - A Phase I/II study of a Ganoderma lucidum extract in patients with Advanced Cancer. Research in Cancer http://ganosecrets.com/section/research/research-in-cancer/.

Zhu HS, Yang XL, Wang LB, Zhao DX, Chen L. 2000 - Effects of extracts from sporodermbroken spores of Ganoderma lucidum on HeLa cells. Cell Biol. Toxicology 16, 20120610.1023/A: 1007663006548.

Zhu XL, Chen AF, Lin ZB. 2007 - Ganoderma lucidum polysaccharides enhance the function of immunological effector cells in immunosuppressed mice. J. Ethnopharmocol 111, $2,21-226$. 